\title{
A TRACE FORMULA FOR SYMMETRIC SPACES
}

\author{
HERVÉ JACQUET, KING F. LAI, AND STEPHEN RALLIS
}

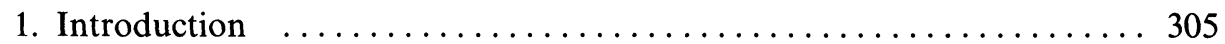

2. The fundamental lemma: unit element $\ldots \ldots \ldots \ldots \ldots \ldots \ldots \ldots \ldots . \ldots . \ldots 312$

3. The fundamental lemma: general element $\ldots \ldots \ldots \ldots \ldots \ldots \ldots \ldots$

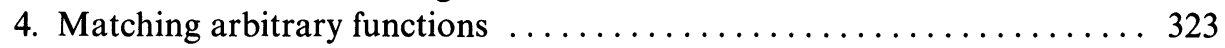

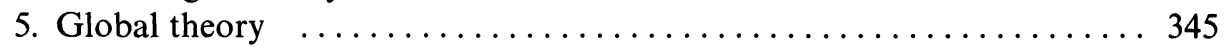

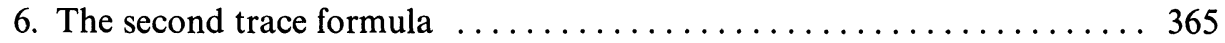

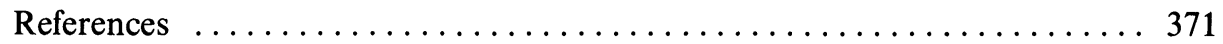

\section{Introduction.}

1.1. Let $G$ be reductive group defined over an algebraically closed field $F$ and let $H$ be the fixator of an involution $\theta$ of $G$. Roughly speaking, the space of double cosets $H \backslash G / H$ is parametrized by the conjugacy classes in another group $G^{\prime}$ (see $[\mathrm{R}],[\mathrm{KR}])$.

In more detail, assume $G$ semisimple and simply connected. Let $\tau$ : $G \rightarrow G$ be the map defined by $\tau(g)=g \theta(g)^{-1}$ and $S$ the image of $\tau$. Clearly, $\tau$ is constant on the left cosets of $G$ modulo $H$ and induces an isomorphism $\sigma$ from $G / H$ to $S$. Then $\sigma(x g H)=x \sigma(g H) x^{-1}$ for $x \in H$. Thus the double cosets of $H \backslash G / H$ correspond via $\sigma$ to the adjoint orbits of $H$ on $S$. The closed $H$-orbits are those of the semisimple elements contained in $S$. They can be described as follows. Let $A$ be a maximal $\theta$-stable torus contained in $S$. All such tori are conjugate under the action of $H$. Then every closed orbit of $H$ in $S$ intersects $A$. Furthermore, there is a Chevalley restriction theorem for the situation at hand. Indeed, let $F[S]$ and $F[A]$ be the rings of regular functions on $S$ and $A$ respectively. Let $H^{0}$ be the neutral connected component of $H$ and let $W_{H}$ be the quotient of the normalizer of $A$ in $H^{0}$ by the centralizer of $A$ in $H^{0}$. Then the restriction map

$$
F[S] \rightarrow F[A]
$$

restricts to an isomorphism

$$
F[S]^{H} \simeq F[A]^{W_{H}} .
$$

Since $F[S]^{H}$ separates the closed orbits, we can identify the set of closed orbits of $H$ in $S$ to the (maximal ideal) spectrum of the algebra $F[A]^{W_{H}}$, that is, to the orbits

Received 27 April 1992. Revision received 23 September 1992.

Jacquet partially supported by NSF grant DMS-91-01637.

Rallis partially supported by NSF grant DMS-91-03263. 
of $W_{H}$ in $A$. Let $R$ be the set of roots of $A$ in $G$; it is a root system. Let $R_{r}$ be the corresponding reduced root system.

Now there is a semisimple group $G^{\prime}$ and a maximal torus $A^{\prime}$ with Weyl group $W^{\prime}$ and root system $R^{\prime}$, and an isomorphism of $A^{\prime}$ onto $A$ which takes $W^{\prime}$ to $W$ and $R^{\prime}$ to $R_{r}$. The usual Chevalley restriction theorem implies that the set of closed $G^{\prime}$-conjugacy classes in $G^{\prime}$ can be identified with the spectrum of

$$
F\left[A^{\prime}\right]^{W^{\prime}}
$$

that is, with the orbits of $W^{\prime}$ in $A^{\prime}$. This gives the required bijection. We note that the isomorphic algebras $F[A]^{W_{H}}$ and $F\left[A^{\prime}\right]^{W^{\prime}}$ are polynomial algebras, but the group $G^{\prime}$ need not be simply connected.

This is the general principle of comparison that guides the particular example to be considered below. Of course, over a global or a local field, it must be modified suitably. Assuming for now its validity over a number field $F$, it is natural to postulate the existence of a trace formula identity of the form

$$
\int K\left(h_{1}, h_{2}\right) d h_{1} d h_{2}=\int_{G^{\prime}(F) \backslash G^{\prime}\left(F_{\mathrm{A}}\right)^{2}} K^{\prime}\left(g^{\prime}, g^{\prime}\right) d g^{\prime} .
$$

Here $K$ and $K^{\prime}$ are the cuspidal kernels, or rather the "discrete parts" of the respective trace formulas. They are associated to functions $f$ and $f^{\prime}$ on $G$ and $G^{\prime}$, respectively; the above equality is supposed to be true if $f$ and $f^{\prime}$ have "matching orbital integrals". The only representations which contribute to the left-hand side are those which are distinguished with respect to $H$, that is, contain a vector $\phi$ such that the integral

$$
\int_{H(F) \backslash H\left(F_{A}\right)} \phi(h) d h
$$

is nonzero. The equality then should characterize distinguished representations as functorial images from representations of $G^{\prime}$.

The purpose of this paper is to explore this idea in a simple case. However, one discovers quickly that such a simple scheme does not work. A more correct formula might take the form

$$
\int K\left(h_{1}, h_{2}\right) \theta\left(h_{1}\right) d h_{1} d h_{2}=\int K^{\prime}\left(g^{\prime}, g^{\prime}\right) d g^{\prime}
$$

or

$$
\int K\left(h_{1}, h_{2}\right) d h_{1} d h_{2}=\int K^{\prime}\left(g^{\prime}, g^{\prime}\right) \theta^{\prime}\left(g^{\prime}\right) d g^{\prime},
$$

where $\theta$ and $\theta^{\prime}$ are suitable automorphic forms on $H$ and $G^{\prime}$ respectively, which serve as "weights" in the formula. 
We now describe the situation at hand. We consider a quadratic extension $E$ of $F$; the group $G$ is the group $G L(2, E)$ regarded as an algebraic group defined over $F$ while $G^{\prime}$ is the group $G L(2, F)$. We denote by $Z$ the center of $G$ (regarded as an algebraic group over $F$ ) and by $Z^{\prime}$ the center of $G^{\prime}$. We also denote by $\eta_{E / F}$ or simply $\eta$ the quadratic character of $F$ attached to $E$. If $E=F(\sqrt{\tau})$, we also write $\eta=\eta_{\tau}$. Let $S^{\prime}$ be the set of invertible hermitian matrices. For every $\varepsilon \in S^{\prime}$ we denote by $H_{\varepsilon}^{0}$ the corresponding unitary group, by $H_{\varepsilon}$ the similitude group, and by $\lambda_{\varepsilon}$ the similitude ratio

$$
{ }^{t} \bar{h} \varepsilon h=\lambda_{\varepsilon}(h) \varepsilon
$$

In particular, for $\varepsilon=w$, where

$$
w=\left(\begin{array}{ll}
0 & 1 \\
1 & 0
\end{array}\right),
$$

we will write simply $H^{0}, H$, and $\lambda$. It will be convenient to introduce the set

$$
S=S^{\prime} w
$$

Thus the group $G L(2, E)$ operates on $S$ by

$$
s \mapsto w^{t} \bar{g} w s g .
$$

It follows that $H$ operates on $S$ as follows:

$$
s \mapsto h^{-1} \operatorname{sh} \lambda(h)
$$

It will be convenient to define a modified action of $H$ on $S$ :

$$
s \mapsto h^{-1} s h .
$$

We now describe a natural map from the set of conjugacy classes of $H$ on $S$ (i.e. the orbits for the modified action) to the set of the conjugacy classes of $G L(2, F)$. We let $S_{0}$ be the intersection of $G L(2, F)$ with $S$; this is the space of matrices in $G L(2, F)$ which are symmetric with respect to the second diagonal. We first recall that all conjugacy classes in $G L(2, F)$ intersect $S_{0}$. More precisely, a semisimple regular element is always conjugate to a matrix in $S_{0}$ of the form

$$
\left(\begin{array}{ll}
\alpha & \beta_{2} \\
\beta_{1} & \alpha
\end{array}\right),
$$

with $\beta_{1} \beta_{2} \neq 0$. Moreover, the conjugacy class of such an element is determined by $\alpha$ and the product $\beta_{1} \beta_{2}$. A unipotent element is always conjugate to an element of 
the form

$$
\left(\begin{array}{ll}
\alpha & \beta \\
0 & \alpha
\end{array}\right), \quad \text { or }\left(\begin{array}{ll}
\alpha & 0 \\
\beta & \alpha
\end{array}\right),
$$

with $\beta \neq 0$. Moreover, the class is determined by $\alpha$. Finally, a central element is always in $S_{0}$. In the case at hand, the comparison principle takes the following form:

LEMma 1.1. Every conjugacy class of $H$ in $S$ intersects $S_{0}$. Moreover, two elements $s_{1}$ and $s_{2}$ in $S_{0}$ are conjugate under $H$ if and only if they are conjugate under $G^{\prime}$.

Proof. We leave the elementary proof to the reader.

1.2. Now we outline the two trace formula identities we have in mind. The action of $G$ on $S$ is not transitive. To compensate for this fact, we consider a set of representatives $\{\varepsilon\}$ for the orbits of $G=G L(2, E)$ on $S^{\prime}$ (or $S$ ); the fixator of $\varepsilon$ in $G$ is then the unitary group $H_{\varepsilon}^{0}$. For each $\varepsilon$ we consider the cuspidal kernel $K_{\varepsilon}$ attached to a function $f_{\varepsilon}$ on $G$ and the trivial central character. On the other hand, the kernel $K^{\prime}$ is the cuspidal kernel attached to a function $f^{\prime}$ on $G^{\prime}$ for the central character $\eta$, except that the contribution of the representations dihedral with respect to $E$ has been removed. The functions $f_{\varepsilon}$ and $f^{\prime}$ are products of local functions which have "matching orbital integrals". At almost all places, the local functions are Hecke functions corresponding to one another by the base-change homomorphism. As usual, part of our task will be to prove the fundamental lemma, i.e. that such Hecke functions indeed have matching orbital integrals.

Recall that $\eta$ is the quadratic character attached to the extension. Recall an automorphic cuspidal representation $\pi$ of $G$ is $H$-distinguished if it contains a vector $\phi$ such that the integral

$$
\int_{H(F) Z\left(F_{A}\right) \backslash H\left(F_{A}\right)} \phi(h) d h
$$

is nonzero. Implicit in this definition is the fact that the central character of the representation $\pi$ is trivial. Similarly, we say that a representation $\pi$ is $\eta$-distinguished if the integral

$$
\int \phi(h) \eta(\lambda(h)) d h
$$

is nonzero for some $\phi$ in the space of $\pi$. Similar definitions apply to the groups $H_{\varepsilon}$. If a representation is distinguished (resp. $\eta$-distinguished) for a group $H_{\varepsilon}$, it is also distinguished (resp. $\eta$-distinguished) with respect to $H$. A proof is outlined in [JY1].

It is known ([HLR]) that an automorphic representation $\pi$ of $G$ with trivial central character is distinguished (resp. $\eta$-distinguished) with respect to $H$ if and only if it is the base change of a representation of $G^{\prime}$ with central character $\eta$ (resp. 
trivial). In particular, $\pi$ cannot be simultaneously distinguished and $\eta$-distinguished. Thus the double integral

$$
\iint K_{\varepsilon}\left(h_{\varepsilon}, h\right) \eta(\lambda(h)) d h_{\varepsilon} d h
$$

is zero. Here the integrals are over the quotients

$$
H_{\varepsilon}(F) Z\left(F_{\AA}\right) \backslash H_{\varepsilon}\left(F_{\AA}\right), H(F) Z\left(F_{\AA}\right) \backslash H\left(F_{\AA}\right) .
$$

In fact, this can be derived from our trace-formula identity. Let $E(h, s)$ be an Eisenstein series on $H$. We normalize it so that it has a pole at $s=1$ with constant residue 1 . It follows that the sum

$$
\sum_{\varepsilon} \iint K_{\varepsilon}\left(h_{\varepsilon}, h\right) E(h, s) \eta(\lambda(h)) d h_{\varepsilon} d h
$$

is actually holomorphic at 1 . Its value at $s=1$ has the form

$$
\sum_{\varepsilon} \int K_{\varepsilon}\left(h_{\varepsilon}, h\right) \theta(h) \eta(\lambda(h)) d h_{\varepsilon} d h
$$

where $\theta$ is defined by

$$
E(h, s)=\frac{1}{s-1}+\theta(h)+O(s-1) .
$$

Our first trace formula is an equality of the form

$$
\sum_{\varepsilon} \int K_{\varepsilon}\left(h_{\varepsilon}, h\right) \theta(h) \eta(\lambda(h)) d h_{\varepsilon} d h=\int K^{\prime}\left(g^{\prime}, g^{\prime}\right) d g^{\prime}
$$

We also consider an Eisenstein series $E^{\prime}(g, s)$ on $G^{\prime}$ with pole at $s=1$. Recall (see [J1]) that for an automorphic representation $\pi^{\prime}$, and for $\phi^{\prime}$ in the space of $\pi^{\prime}$, the integral

$$
\int \phi^{\prime}(g) \overline{\phi^{\prime}}(g) E^{\prime}(g, s) \eta(\operatorname{det}(g)) d g
$$

is an holomorphic multiple of the $L$-function

$$
L\left(s, \pi^{\prime} \otimes \eta \times \tilde{\pi}^{\prime}\right) .
$$

In particular, if $\pi^{\prime}$ is not a dihedral representation for the extension $E$, this $L$-function 
is holomorphic at $s=1$. The integral

$$
\int K^{\prime}(g, g) \eta(\operatorname{det} g) E^{\prime}(g, s) d g
$$

is thus holomorphic at $s=1$. Its value has the form

$$
\int K^{\prime}(g, g) \theta^{\prime}(g) d g
$$

where $\theta^{\prime}$ is defined in the same way as $\theta$. Our second trace-formula identity has the form

$$
\sum_{\varepsilon} \int K_{\varepsilon}\left(h_{\varepsilon}, h\right) d h_{\varepsilon} d h=\int K^{\prime}\left(g^{\prime}, g^{\prime}\right) \theta^{\prime}\left(g^{\prime}\right) d g^{\prime}
$$

We timorously conjecture that similar identities are true for any symmetric space.

1.3. The case at hand has a special added feature. Indeed, we will derive our identities by taking residues in identities valid for all values of a complex parameter s. They read

$$
\begin{gathered}
\left|D_{E}\right|^{1 / 2}\left|D_{F}\right|^{-1} L\left(s, 1_{F}\right) \sum_{\varepsilon} \int K\left(h_{\varepsilon}, h\right) d h_{\varepsilon} E(h, s) \eta(\lambda(h)) d h \\
=L(s, \eta) \int K^{\prime}\left(g^{\prime}, g^{\prime}\right) E^{\prime}\left(g^{\prime}, s\right) d g^{\prime}
\end{gathered}
$$

and

$$
\begin{aligned}
& L(s, \eta) \sum_{\varepsilon} \int K\left(h_{\varepsilon}, h\right) d h_{\varepsilon} E(h, s) d h \\
& \quad=\left|D_{E}\right|^{1 / 2}\left|D_{F}\right|^{-1} L\left(s, 1_{F}\right) \int K^{\prime}\left(g^{\prime}, g^{\prime}\right) E^{\prime}\left(g^{\prime}, s\right) \eta\left(\operatorname{deg} g^{\prime}\right) d g^{\prime}
\end{aligned}
$$

Here $D_{E}$ and $D_{F}$ are the respective discriminants. The integrals converge absolutely for $\mathfrak{R} s>1$ and have meromorphic continuation. The kernels $K_{\varepsilon}$ and $K^{\prime}$ correspond to functions with "matching orbital integrals", the matching being uniform in $s$. The precise form of our identities will be given in the text.

Let us remark that the right-hand sides of our identities are the kind of integrals considered in [JZ]. We would like to think of them as the Zagier trace formula. The left-hand sides are thus a "relative version" of the Zagier trace formula. The above identity equality involving the Zagier trace formula and the relative version of the Zagier trace formula is directly related to a similar equality involving the RankinSelberg $L$-function for $G L(2)$ and the $L$-function studied by Asai ([A]). In turn, this 
relation is the key to the characterization of the distinguished representations given in [HLR]. We do not expect to have a similar identity - valid for all $s$-for other symmetric spaces.

Another approach to the study of distinguished representations is an identity of the form

$$
\sum_{\varepsilon} \int K\left(h_{\varepsilon}, n\right) d h_{\varepsilon} \theta(n) d n=\int K^{\prime}\left(n_{1}^{\prime}, n_{2}^{\prime}\right) \theta^{\prime}\left(n_{1}^{\prime} n_{2}^{\prime}\right) d n_{1}^{\prime} d n_{2}^{\prime}
$$

Here $N$ and $N^{\prime}$ are the groups of upper triangular matrices with unit diagonals in $G$ and $G^{\prime}$ respectively; $\theta$ and $\theta^{\prime}$ are characters of $N\left(F_{\mathbb{A}}\right)$ and $N^{\prime}\left(F_{\mathbb{A}}\right)$ trivial on the rational points, and the integrals are over

$$
N(F) \backslash N\left(F_{\mathbb{A}}\right)^{2}, N^{\prime}(F) \backslash N^{\prime}\left(F_{\mathbb{A}}\right)^{2}
$$

respectively. See [JY1] for more details. In principle, this formula can be obtained from our second identity by taking the limit as $s$ tends to infinity. This identity was discovered first and should generalize to symmetric spaces where $G$ is quasi-split. It provides information which is different from the information provided by the formula considered here. For more information on this approach, see [F], [I1], [I2], $[\mathrm{J} 2],[\mathrm{J} 3],[\mathrm{JR}],[\mathrm{M}],[\mathrm{Y}],[\mathrm{JY} 1],[\mathrm{JY} 2]$, and [Z]. The present approach seems to be more geometric and to introduce the weight factors $\theta$ which appear as an essential ingredient of the situation.

We also mention another class of problems for symmetric spaces. Let $S$ be the symmetric space of $s \in G$ such that $s \bar{s}=1$. Now let $G_{1}$ be an inner form of $G L(2, E)$ which is defined over $F$. Consider the symmetric space $S_{1}$ of $s_{1} \in G_{1}$ such that $s_{1} \bar{s}_{1}=1$, where $\overline{s_{1}}$ means the conjugate of $s_{1}$ for the Galois action which defines $G_{1}$ over $F$. We may think of $S_{1}$ as being an inner form of $S$ and compare distinguished representations for $G$ and $G_{1}$. This was done in [JL] under restrictive assumptions necessitated by the lack of local information. The purpose of Hakim's thesis was to provide the missing local information ([Ha]). The global application follows easily. It is described in reference $[\mathrm{FH}]$ which is largely "based on Hakim's thesis". One expects comparison results between a symmetric space and its inner forms to be valid under much more general assumptions (see $[F]$ for the case of $G L(n)$ ).

1.4. The material is arranged as follows. We begin with the first trace formula. We prove the fundamental lemma for the unit element of the Hecke algebra in Section 2, and for the general element in Section 3. We establish that we can match arbitrary functions in Section 4; in Section 5 we prove our trace formula under some restrictive assumptions. Finally, in Section 6 we indicate how to modify our arguments to obtain the second trace formula. For simplicity, we prove our formula under restrictive assumptions specified in the text.

In the present work, we consider only representations of $G$ with trivial central character. One should, more generally, consider representations of $G$ with a central 
character which is the base change of a character of $F$. Moreover, in the present work, we have singled out a unitary group $H$, namely the split one. One should also investigate an identity where one unitary group other than the split one is singled out. We intend to come back to these questions in a subsequent work.

\section{The fundamental lemma: unit element.}

2.1. We consider an unramified quadratic extension $E / F$ of local fields with odd residual characteristic. We let $\eta_{E / F}$ or $\eta$ be the quadratic character of $F$ attached to $E$. We denote by $R_{F}$ (respectively $R_{E}$ ) the ring of integers of $F$ (resp. $E$ ), by $P_{F}$ (respectively $P_{E}$ ) the maximal ideal in that ring. We denote by $q_{F}$ or simply $q$ the cardinality of $R_{F} / P_{F}$. Thus $R_{E} / P_{E}$ has $q^{2}$ elements. We denote by $\alpha_{F}$ the module function on $F$.

We let $P$ be the group of upper triangular matrices in $G L(2, E)$, regarded as a group defined over $F$, and let $N$ be its unipotent radical. We set

$$
\begin{gathered}
P^{\prime}=P \cap G^{\prime}, \quad N^{\prime}=N \cap G^{\prime}, \quad P_{H}=P \cap H, \quad N_{H}=N \cap H, \\
K=G L\left(2, R_{E}\right), \quad K^{\prime}=G L\left(2, R_{F}\right),
\end{gathered}
$$

and we let $f(h, s)$ be the function on $H$ defined by

$$
f(h, s)=|a|^{s}, \quad \text { if } h=\left(\begin{array}{ll}
1 & x \\
0 & 1
\end{array}\right) z\left(\begin{array}{ll}
a & 0 \\
0 & 1
\end{array}\right) k
$$

with $k \in K \cap H, a \in F^{\times}, x+\bar{x}=0$ and $z \in Z$. We let $\Phi$ be a smooth function of compact support on $S$. For $\sigma$ in $S_{0}$ but not in $P^{\prime}$, we set

$$
\Omega(\Phi, \sigma, s)=\int \Phi\left(h^{-1} z \sigma h\right) \eta(\lambda(h)) f(h, s) d h d^{\times} z .
$$

The integral in $h$ is over $Z \backslash H$, the measure to be specified later, the integral in $z$ is over $Z^{\prime} \simeq F^{\times}$. It will be proved below that the integral converges absolutely for $\mathfrak{R} s>0$.

On the other hand, let $\Phi^{\prime}$ be a smooth function of compact support on $G^{\prime}$. We set

$$
\Omega^{\prime}\left(\Phi^{\prime}, \sigma, s\right)=\int \Phi^{\prime}\left(g^{\prime-1} z \sigma g^{\prime}\right) f^{\prime}\left(g^{\prime}, s\right) d g^{\prime} \eta(z) d^{\times} z,
$$

where $f^{\prime}\left(g^{\prime}, s\right)$ is the function on $G L(2, F)$ defined in the same way as $f$. The integral is over $G^{\prime} / Z^{\prime}$ and $Z^{\prime} \simeq F^{\times}$. It converges for $\mathfrak{R} s>0$.

We shall say $\Phi$ and $\Phi^{\prime}$ have matching orbital integrals if

$$
\Omega(\Phi, \sigma, s) L\left(s, 1_{F}\right)=\eta\left(\beta_{1}\right) \Omega^{\prime}\left(\Phi^{\prime}, \sigma, s\right) L(s, \eta)
$$


for all $s$ with $\mathfrak{R} s>0$ and all $\sigma$ of the form

$$
\sigma=\left(\begin{array}{cc}
\alpha & \beta_{2} \\
\beta_{1} & \alpha
\end{array}\right)
$$

with $\beta_{1} \beta_{2} \neq 0$. The measures are chosen as follows. We choose a $\tau_{0}$ such that $E=F\left(\sqrt{\tau_{0}}\right)$. Then conjugacy by

$$
\left(\begin{array}{cc}
\sqrt{\tau_{0}} & 0 \\
0 & 1
\end{array}\right)
$$

transforms $H$ into the group $G L(2, F) Z$, and we use this map to transport the Haar measure on $H / Z$ to

$$
G L(2, F) Z / Z \simeq G L(2, F) / Z^{\prime}
$$

Thus the measure on $H / Z$ depends only on the measure on $G^{\prime} / Z^{\prime}$.

To prove the convergence of the integrals we proceed as follows. Suppose that $\mathfrak{R} s>0$. Then we can write

$$
L\left(2 s, 1_{F}\right) f^{\prime}(g, s)=\int \Phi_{0}[(0, x) g]|x|^{2 s} d^{\times} x|\operatorname{deg} g|^{s}
$$

where $\Phi_{0}$ is the characteristic function of $R_{F}^{2}$. Let $\sigma$ be an element of $S_{0}$ such that $\beta_{1} \beta_{2} \neq 0$. We denote by $T^{\prime}$ the torus of $G^{\prime}$ which centralizes $\sigma$. It can be viewed as the multiplicative group of a commutative semisimple algebra $L^{\prime}$. Then

$$
L(2 s, 1) \Omega^{\prime}\left(\Phi^{\prime}, \sigma, s\right)=\int_{T^{\prime} \backslash G^{\prime}} \mu\left(g^{\prime}\right) d g^{\prime} \int \Phi_{0}\left[(0,1) t^{\prime} g^{\prime}\right]\left|\operatorname{det} t^{\prime}\right|^{s} d t^{\prime}
$$

where

$$
\mu\left(g^{\prime}\right)=\int \Phi^{\prime}\left(g^{\prime-1} z \sigma g^{\prime}\right) \eta(z) d^{\times} z
$$

The inner integral is actually a Tate integral for the algebra $L^{\prime}$. Thus it converges absolutely for $\Re s>0$. On the other hand, $\mu$ is, for fixed $\sigma$, a smooth function of compact support on $T^{\prime} \backslash G^{\prime}$. This implies that $\Omega^{\prime}$ converges for $\Re s>0$. Our argument also shows that, for each $s, \Omega^{\prime}\left(\Phi^{\prime}, \sigma, s\right)$ is a smooth function on the set of $\sigma$ such that $\beta_{1} \beta_{2} \neq 0$. We can use the isogeny (4) to transport the integral $\Omega$ to the group $G^{\prime}$ and obtain an integral whose convergence is established in a similar way.

A formal computation shows that relation (3) is satisfied for all $\left(\alpha, \beta_{1}, \beta_{2}\right)$ with $\beta_{1} \beta_{2} \neq 0$ if it is satisfied for $\alpha \in\{0,1\}, \beta_{1}=1$, and $\beta_{2} \neq 0$. We will write $\Omega(\Phi, \beta, s)$ 
and $\Omega^{\prime}\left(\Phi^{\prime}, \beta, s\right)$ for the integrals when

$$
\sigma=\left(\begin{array}{ll}
1 & \beta \\
1 & 1
\end{array}\right)
$$

Similarly, we will write $\Omega^{\infty}(\Phi, \beta, s)$ and $\Omega^{\prime \infty}\left(\Phi^{\prime}, \beta, s\right)$ for the integrals when

$$
\sigma=\left(\begin{array}{ll}
0 & \beta \\
1 & 0
\end{array}\right)
$$

Let

$$
\sigma=\left(\begin{array}{ll}
\alpha & \beta \\
1 & \alpha
\end{array}\right)
$$

Then

$$
\Omega(\Phi, \sigma, s)=|\alpha|^{s} \eta(\alpha) \Omega\left(\Phi, \beta \alpha^{-2}, s\right)
$$

If we fix $\beta$ and let $\alpha$ tend to 0 , we obtain, for $|\alpha|$ small enough,

$$
\Omega^{\infty}(\Phi, \beta, s)=|\alpha|^{s} \eta(\alpha) \Omega\left(\Phi, \beta \alpha^{-2}, s\right)
$$

A similar relation is true for $\Omega^{\prime}$. Thus we see that the relation

$$
\Omega(\Phi, \beta, s) L\left(s, 1_{F}\right)=\Omega^{\prime}\left(\Phi^{\prime}, \beta, s\right) L(s, \eta)
$$

for all $\beta$ implies that the functions have matching orbital integrals.

Proposition 2.1. Assume the extension is unramified of odd residual characteristic. Let $\Phi_{0}$ be the characteristic function of $K \cap S$ and let $\Phi_{0}^{\prime}$ be the characteristic function of $K^{\prime}$. Then $\Phi_{0}$ and $\Phi_{0}^{\prime}$ have matching orbital integrals.

2.2. The proof of the proposition is based on the computation of an integral that we now introduce:

$$
A(t, s)=\int_{|x| \leqslant 1} \frac{1-\left|t-x^{2}\right|^{s-1} q^{-s+1}}{1-q^{-s+1}} d x
$$

Here the volume of $R_{F}$ is one. We let $q$ be the cardinality of the residual field of $F$ and write $A(t, X)$ for $A(t, s)$ if $X=q^{-s}$. We let $\tau_{0}$ be any unit of $F$ which is not a square in $E$, and we let $\varpi$ be a uniformizer. 
Lemma 2.2. Let $t$ be an integer. Then $A(t, s)$ converges absolutely for $\mathfrak{R} s>0$. It is given by the formulas, where $X=q^{-s},|y| \leqslant 1$,

$$
A(t, s)= \begin{cases}\frac{1+X}{1-X^{2} q}+|y|^{2 s-1} \frac{X(1+X)(1-X q)}{\left(1-X^{2} q\right)(1-X)} & \text { if } t=y^{2} \\ \frac{1+X}{1-X^{2} q}-\frac{|y|^{2 s-1} X(1+X q)}{1-X^{2} q} & \text { if } t=y^{2} \tau_{0} \\ \frac{1+X}{1-X^{2} q}-\frac{|y|^{2 s-1} X^{2} q(1+X)}{1-X^{2} q} & \text { if } t=y^{2} \varpi .\end{cases}
$$

We leave the computation to the reader.

The following lemma is then easily verified.

LEMMA 2.3. If $\tau_{0}$ is any unit which is not a square, then

$$
\begin{aligned}
\frac{A\left(y^{2},-X\right)}{1-X} & =\frac{A\left(y^{2} \tau_{0}, X\right)}{1+X} \\
\frac{A\left(y^{2}, X\right)}{1+X} & =\frac{A\left(y^{2} \tau_{0},-X\right)}{1-X} .
\end{aligned}
$$

If $\varpi$ and $\varpi^{\prime}$ are two uniformizers, then

$$
\frac{A\left(y^{2} \varpi,-X\right)}{1-X}=\frac{A\left(y^{2} \varpi^{\prime}, X\right)}{1+X}
$$

2.3. We now compute the orbital integrals for

$$
\sigma=\left(\begin{array}{ll}
1 & \beta \\
1 & 1
\end{array}\right)
$$

where $\beta$ is nonzero. We compute first $\Omega$. We set

$$
h=\left(\begin{array}{ll}
1 & x \\
0 & 1
\end{array}\right)\left(\begin{array}{ll}
t & 0 \\
0 & 1
\end{array}\right) k .
$$

Then

$$
d h=d u|t|^{-1} d^{\times} t d k
$$


if $x=\sqrt{\tau_{0}} u$ and $\tau_{0}$ is a unit which is not a square. The measure of $R_{F}$ for $d u$ is one, and the measure of $R^{\times}$for $d^{\times} t$ is also one. In passing, we remark that our choice of measures suggests writing $x=u / \sqrt{\tau_{0}}$ instead. Since $\tau_{0}$ is a unit, the measure $d x$ is the same. However, when $E$ is ramified, we will take $\tau_{0}$ to be a uniformizer and will write $x=u / \sqrt{\tau_{0}}$. Now

$$
f(h, s)=|t|^{s}
$$

We get

$$
\Omega\left(\Phi_{0}, \beta, s\right)=\int \Phi_{0}\left[\left(\begin{array}{cc}
z(1-x) & t^{-1} z\left(\beta-x^{2}\right) \\
z t & z(1+x)
\end{array}\right)\right]|t|^{s-1} \eta(t) d^{\times} t d u d^{\times} z
$$

The integral is zero unless $1-\beta$ is a norm. Assuming this to be the case, we set

$$
|1-\beta|=|\gamma|^{2}
$$

The support of the integrand is defined by the conditions

$$
\begin{gathered}
\left|(1-\beta) z^{2}\right|=1, \quad|z| \leqslant 1, \quad|z x| \leqslant 1, \\
\left|z\left(\beta-x^{2}\right)\right| \leqslant|t| \leqslant|z|^{-1} .
\end{gathered}
$$

Integrating with respect to $t$ first, we get

$$
\int \frac{\eta(z)|z|^{1-s}+|z|^{s-1} \eta(z)\left|\beta-x^{2}\right|^{s-1} \eta\left(\beta-x^{2}\right) q^{-s+1}}{1+q^{-s+1}} d u d^{\times} z .
$$

Integrating over $z$, we get

$$
\eta(\gamma) \int_{|u| \leqslant|\gamma|} \frac{|\gamma|^{s-1}+|\gamma|^{1-s}\left|\beta-x^{2}\right|^{s-1} \eta\left(\beta-x^{2}\right) q^{-s+1}}{1+q^{-s+1}} d u
$$

Recall $x=\sqrt{\tau_{0}} u$. We now change $u$ to $u \gamma$. We arrive at the expression

$$
|\gamma|^{s} \eta(\gamma) \int_{|u| \leqslant 1} \frac{1+\eta\left(\frac{\beta}{\gamma^{2}}-x^{2}\right)\left|\frac{\beta}{\gamma^{2}}-x^{2}\right|^{s-1} q^{-s+1}}{1+q^{-s+1}} d u .
$$

Thus

$$
\Omega\left(\Phi_{0}, \sigma, s\right)=|\gamma|^{s} \eta(\gamma) \int_{|u| \leqslant 1} \frac{1+\eta\left(t-u^{2}\right)\left|t-u^{2}\right|^{s-1} q^{-s+1}}{1+q^{-s+1}} d u
$$


where

$$
t=\frac{\beta}{\gamma^{2} \tau_{0}}
$$

We remark that $t$ is always an integer. Finally,

$$
\Omega\left(\Phi_{0}, \beta, s\right)=|\gamma|^{s} \eta(\gamma) A\left(\frac{\beta}{\gamma^{2} \tau_{0}},-X\right)
$$

2.4. We pass to the computation of the integral $\Omega^{\prime}$. We set

$$
g=\left(\begin{array}{ll}
1 & x \\
0 & 1
\end{array}\right)\left(\begin{array}{ll}
t & 0 \\
0 & 1
\end{array}\right) k
$$

Then

$$
d g=d x|t|^{-1} d^{\times} t d k, \quad f^{\prime}(g, s)=|t|^{s} .
$$

As before, we find

$$
\Omega^{\prime}\left(\Phi_{0}^{\prime}, \beta, s\right)=\int \Phi_{0}^{\prime}\left[\left(\begin{array}{cc}
z(1-x) & t^{-1} z\left(\beta-x^{2}\right) \\
z t & z(1+x)
\end{array}\right)\right]|t|^{s-1} d^{\times} t d x \eta(z) d^{\times} z .
$$

However, this time $x$ is in $F$. As before, the integral is zero unless

$$
|1-\beta|=|\gamma|^{2}
$$

and the support of the integrand is defined by

$$
\begin{gathered}
\left|(1-\beta) z^{2}\right|=1, \quad|z| \leqslant 1, \quad|z x| \leqslant 1 \\
\left|z\left(\beta-x^{2}\right)\right| \leqslant|t| \leqslant|z|^{-1} .
\end{gathered}
$$

Integrating with respect to $t$ first, we get

$$
\int \frac{|z|^{-s+1}-|z|^{s-1}\left|\beta-x^{2}\right|^{s-1} q^{-s+1}}{1-q^{-s+1}} d x \eta(z) d^{\times} z .
$$

Integrating over $z$, we get

$$
\Omega^{\prime}\left(\Phi_{0}^{\prime}, \sigma, s\right)=\eta(\gamma) \int \frac{|\gamma|^{s-1}-|\gamma|^{1-s}\left|\beta-x^{2}\right|^{s-1} q^{-s+1}}{1-q^{-s+1}} d x
$$


the integral over $|x| \leqslant|\gamma|$. If we change $x$ to $x \gamma$, we arrive at

$$
|\gamma|^{s} \eta(\gamma) \int_{|x| \leqslant 1} \frac{1-\left|t-x^{2}\right|^{s-1} q^{-s+1}}{1-q^{-s+1}} d x
$$

with $t=\beta / \gamma^{2}$, that is,

$$
\Omega^{\prime}\left(\Phi_{0}^{\prime}, \beta, s\right)=|\gamma|^{s} \eta(\gamma) A\left(\frac{\beta}{\gamma^{2}}, X\right)
$$

Comparing with Lemma (2.3), we get equation (5) and the proposition.

\section{The fundamental lemma: general element.}

3.1. We keep the notations of the previous section. Let $f$ be a bi-K-invariant on $G$ of compact support and $f^{\prime}$ be a bi-K-invariant function on $G^{\prime}$ with compact support. We shall say that $f^{\prime}$ corresponds to $f$ if the following condition is satisfied. For $x=q^{-s}$ set $X=q^{-2 s}$; let $\Pi$ be the unramified component of the representation of $G$ induced by $\left(\alpha_{E}^{s}, \alpha_{E}^{-s}\right)$ and let $\pi$ be the unramified component of the representation of $G^{\prime}$ induced by $\left(\alpha_{F}^{s}, \eta \alpha_{F}^{-s}\right)$. Thus $\Pi$ is the base change of $\pi$, and for any $x$,

$$
\operatorname{Tr} \Pi(f)=\operatorname{Tr} \pi\left(f^{\prime}\right)
$$

We note that this relation makes sense if $f$ is invariant under the center of $G$ and $f^{\prime}$ transforms under the character $\eta$ of the center of $G^{\prime}$. If it is satisfied, we shall also say that $f^{\prime}$ corresponds to $f$.

Let $\Phi_{0}$ be the characteristic function of $K \cap S^{\prime}$. We define a function $\Phi_{f}$ on $S^{\prime}$ by

$$
\Phi_{f}(s)=\int_{H^{0}} f(h g) d h
$$

if $s$ is of the form $s={ }^{t} \bar{g} w g$, and

$$
\Phi_{f}(s)=0
$$

if $s$ is not in the orbit of $w$. We assume that the measure of

$$
H^{0} \cap K
$$

is one. If $f_{0}$ is the characteristic function of $K$, then $\Phi_{f_{0}}=\Phi_{0}$. In general, since $f=f_{0} * f$, we can write

$$
\Phi_{f}(s)=\int \Phi_{0}\left({ }^{t} \bar{g} s g\right) f\left(g^{-1}\right) d g .
$$


The bijection $s \mapsto s w$ allows us to identify $S^{\prime}$ to $S$ and to regard a function $\Phi$ on $S^{\prime}$ as a function on $S$ that we denote also by $\Phi$. We want to prove the following statement.

Proposition 3.1. If $f^{\prime}$ corresponds to $f$, then the functions $\Phi_{f}$ and $f^{\prime}$ have matching orbital integrals.

3.2. We need some preliminaries. For almost all $X$, the representation $\Pi$ introduced above is $H$-distinguished in the sense that there is a nonzero linear $\mu$ form on the space of $\Pi$ such that

$$
\mu(\Pi(h) v)=\mu(v),
$$

for every vector $v$ in the space of $\Pi$ and $h \in H$. Let $v_{0}$ be a $K$-fixed vector. Then the function $\omega$ defined by

$$
\omega(g)=\mu\left(\Pi(g) v_{0}\right)
$$

is $H$-invariant on the left and an eigenfunction of the Hecke operators on the right. In particular, let $f_{1}$ be the characteristic function of

$$
K\left(\begin{array}{ll}
\varpi & 0 \\
0 & 1
\end{array}\right) K
$$

where $\varpi$ is a uniformizer. Then

$$
\int \omega(g y) f_{1}(y) d y=\left(X+X^{-1}\right) q \omega(g) .
$$

Since $\omega$ is invariant under the center of $G$, this relation implies conversely that $\omega$ is an eigenfunction of the Hecke operators. Next, let $\Omega$ be the function on $S^{\prime}$ defined by

$$
\Omega(s)=\omega(g)
$$

if $s={ }^{t} \bar{g} w g$ for some $g$, and

$$
\Omega(s)=0
$$

otherwise. Then

$$
\Omega(s z)=\Omega(s)
$$

for $z \in F^{\times}$because the image of $H$ under the similitude ratio map is $F^{\times}$. Moreover, for all $k \in K$,

$$
\Omega\left({ }^{t} \bar{k} s k\right)=\Omega(s)
$$


and

$$
\int \Omega\left({ }^{t} \bar{g} s\right) f_{1}(g) d g=\left(X+X^{-1}\right) q \Omega(s)
$$

We will show that for all $X$ there is a function $\Omega$ satisfying conditions (10)-(13), unique within a constant factor; moreover $\Omega(e) \neq 0$.

The theory of elementary divisors shows that a set of representatives for the orbits of $K$ on $S^{\prime}$ is the set of matrices of the form

$$
\left(\begin{array}{cc}
\varpi^{i} & 0 \\
0 & \varpi^{j}
\end{array}\right)
$$

with $i \geqslant j$. Moreover, such a matrix is in the orbit of $w$ if and only if its determinant is minus a norm, that is, $i+j$ is even. In particular, the function $\Omega$ is determined by its values on the above matrices with $i=2 n, j=0$. We denote these values by $\Omega_{n}$. We denote by $P_{E}$ the maximal ideal in $R_{E}$. Then the above equation is equivalent to the difference equation

$$
\begin{gathered}
\Omega_{1}+\left(q^{2}-q-1\right) \Omega_{1}+(q+1) \Omega_{0}=\left(X+X^{-1}\right) q \Omega_{0}, \\
\Omega_{n-1}+q^{2} \Omega_{n+1}=\left(X+X^{-1}\right) q \Omega_{n}, \quad \text { if } n>0 .
\end{gathered}
$$

Clearly, these conditions determine the scalars $\Omega_{n}$ uniquely in terms of $\Omega_{0}$. In particular, we can take $\Omega_{0}=1$. This shows the existence and uniqueness of $\Omega$. We will denote by $\Omega_{X}$ the function we just defined. Solving the difference equation, we find

$$
\Omega_{n}=\frac{q X-1}{(X+1)(q-1)}\left(X q^{-1}\right)^{n}+\frac{q X^{-1}-1}{\left(X^{-1}+1\right)(q-1)}\left(X^{-1} q^{-1}\right)^{n}
$$

To continue, there is an invariant measure on the orbit of $w$ under $G$. This measure can be computed as follows

$$
\int \Phi(s) d s=\sum_{i \geqslant j} \int \Phi\left({ }^{t} \bar{k}\left(\begin{array}{cc}
\varpi^{i} & 0 \\
0 & \varpi^{j}
\end{array}\right) k\right) d k \gamma\left(\varpi^{i-j}\right)
$$

To compute $\gamma$ we write that

$$
\int \Phi\left({ }^{t} \bar{g} s g\right) f_{1}(g) d g d s=\int f_{1}(g) d g \int \Phi(s) d s
$$


We take the measure of $K \cap S$ to be 1 so that $\gamma(1)=1$. Applying the previous relation to the characteristic function of an orbit of $K$, we find a recurrence relation for $\gamma\left(\varpi^{2 m}\right)$, and then

$$
\gamma(1)=1, \quad \gamma\left(\varpi^{2 m}\right)=q^{2 m}\left(1-q^{-1}\right) \quad \text { for } m>0 .
$$

We also denote by $\omega_{x}$ the spherical function attached to the representation $\pi$ and by $\omega_{n}$ its value on the matrix

$$
\left(\begin{array}{cc}
\varpi^{n} & 0 \\
0 & 1
\end{array}\right)
$$

Recall this is

$$
\omega_{n}=\frac{q x^{2}+1}{(q+1)\left(x^{2}+1\right)}\left(x q^{-1 / 2}\right)^{n}+\frac{q x^{-2}+1}{(q+1)\left(x^{-2}+1\right)}\left(-x^{-1} q^{-1 / 2}\right)^{n}
$$

Of course, this can be proved as before by solving a difference equation. We also recall that the Haar measure on $G^{\prime}$ can be computed like so:

$$
\int f^{\prime}\left(g^{\prime}\right) d g^{\prime}=\sum_{i \geqslant j} \iint f^{\prime}\left(k_{1}\left(\begin{array}{cc}
\varpi^{i} & 0 \\
0 & \varpi^{j}
\end{array}\right) k_{2}\right) d k_{1} d k_{2} \gamma^{\prime}\left(\varpi^{i-j}\right),
$$

where $\gamma^{\prime}$ is defined by

$$
\gamma^{\prime}(1)=1, \quad \gamma^{\prime}\left(\varpi^{m}\right)=q^{m}\left(1+q^{-1}\right) \quad \text { for } m>0 .
$$

Recall that $X=x^{2}$. It is now a simple matter to verify the following relations:

LemMA 3.2. Notation being as above, for any $n \geqslant 1$,

$$
\begin{aligned}
\Omega_{n} \gamma\left(\varpi^{2 n}\right)+\Omega_{n-1} \gamma\left(\varpi^{2(n-1)}\right) & =\omega_{2 n} \gamma^{\prime}\left(\varpi^{2 n}\right)-\omega_{2(n-1)} \gamma^{\prime}\left(\varpi^{2(n-1)}\right), \\
\sum_{0 \leqslant i \leqslant n} \Omega_{n-i} \gamma\left(\varpi^{2(n-i)}\right) & =\sum_{0 \leqslant i \leqslant n} \omega_{2(n-i)} \gamma^{\prime}\left(\varpi^{2(n-i)}\right)(-1)^{i} .
\end{aligned}
$$

3.3. Now suppose that $f$ and $f^{\prime}$ correspond to one another. Then equality (9) gives

$$
\int \Omega_{X}\left({ }^{t} \bar{g} w g\right) f(g) d g=\int \omega_{x}\left(g^{\prime}\right) f^{\prime}\left(g^{\prime}\right) d g^{\prime}
$$

In terms of the function $\Phi=\Phi_{f}$ on $S^{\prime}$ associated to $f$, this reads

$$
\int \Omega_{X}(s) \Phi(s) d s=\int \omega_{x}\left(g^{\prime}\right) f^{\prime}\left(g^{\prime}\right) d g^{\prime}
$$


Conversely, if this relation is satisfied, then $f^{\prime}$ is associated to $f$. If $\Phi$ is a $K$-invariant function of compact support on $S^{\prime}$ with support in the orbit of $w$ and $f^{\prime}$ is a Hecke function on $G^{\prime}$, we shall say that $f^{\prime}$ corresponds to $\Phi$ if this relation is satisfied for all $x$. To prove the proposition it will suffice to show that, given $\Phi$ and a corresponding $f^{\prime}$, then $\Phi$ and $f^{\prime}$ have matching orbital integrals.

Now the previous lemma and the integration formulas imply the following lemma:

LEMMA 3.3. Let $\Phi_{n}$ be the characteristic function of the set of matrices $s$ in $S^{\prime}$ with integral entries and $|\operatorname{det} s|=q^{-2 n}$. Let $\Phi_{n}^{\prime}$ be similarly the characteristic function of the set of matrices $g^{\prime}$ in $G^{\prime}$ with integral entries and $\left|\operatorname{deg} g^{\prime}\right|=q^{-n}$. Then $\Phi_{n}$ corresponds to $\Phi_{2 n}^{\prime}$.

Proof. Indeed, the equality to be proved reads

$$
\int \Phi_{n}(s) \Omega_{X}(s) d s=\int \Phi_{2 n}^{\prime}\left(g^{\prime}\right) \omega_{x}\left(g^{\prime}\right) d g^{\prime}
$$

and follows at once from the previous lemma and the formulas for $\gamma$ and $\gamma^{\prime}$.

Now the translates of $\Phi_{n}$ under the center form a basis of the space of $K$-invariant functions on $S^{\prime}$ (or $S$ ) with support in the set of matrices with determinant of even valuation. We see that the proposition will be completely proved if we prove the following lemma:

LEMMA 3.4. For $n \geqslant 0$ the functions $\Phi_{n}$ and $\Phi_{2 n}^{\prime}$ have matching orbital integrals.

Proof. We regard $\Phi_{n}$ as a function on $S$. We first compute the orbital integral of $\Phi_{n}$ with $n \geqslant 0$. We proceed as before: the integral is equal to

$$
\Omega\left(\Phi_{n}, \beta, s\right)=\int|t|^{s-1} \eta(t) d^{\times} t d^{\times} z d u
$$

where $x=u \sqrt{\tau_{0}}$ and the integral is over the domain

$$
\begin{gathered}
\left|(1-\beta) z^{2}\right|=q^{-2 n}, \\
|z| \leqslant 1, \quad|z x| \leqslant 1, \\
\left|z\left(\beta-x^{2}\right)\right| \leqslant|t| \leqslant|z|^{-1} .
\end{gathered}
$$

The integral is zero unless

$$
|1-\beta|=|\gamma|^{2}, \quad|\gamma| q^{n} \geqslant 1 .
$$

Assuming this condition is satisfied, we find

$$
\Omega\left(\Phi_{n}, \beta, s\right)=|\gamma|^{s} q^{n s} \eta(\gamma)(-1)^{n} A\left(\frac{\beta \varpi^{2 n}}{\gamma^{2} \tau_{0}},-X\right)
$$

where $A$ was defined in the previous section. 
We pass to the computation of $\Omega^{\prime}\left(\Phi_{2 n}^{\prime}, \beta, s\right)$. We proceed as before: the integral is equal to

$$
\Omega^{\prime}\left(\Phi_{2 n}^{\prime}, \beta, s\right)=\int|t|^{s-1} \eta(z) d^{\times} t d^{\times} z d x
$$

taken over

$$
\begin{gathered}
\left|(1-\beta) z^{2}\right|=q^{-2 n}, \\
|z| \leqslant 1, \quad|z x| \leqslant 1, \\
\left|z\left(\beta-x^{2}\right)\right| \leqslant|t| \leqslant|z|^{-1} .
\end{gathered}
$$

We see the integral is zero unless

$$
|1-\beta|=|\gamma|^{2}, \quad|\gamma| q^{n} \geqslant 1
$$

Assuming this condition is satisfied, we find

$$
\Omega^{\prime}\left(\Phi_{2 n}^{\prime}, \beta, s\right)=|\gamma|^{s} q^{n s} \eta(\gamma)(-1)^{n} A\left(\frac{\beta \varpi^{2 n}}{\gamma^{2}}, X\right)
$$

Comparing the above explicit formulas and the functional equation of the function $A$, we see that our functions have indeed matching orbital integrals. This concludes the proof of the lemma and the proposition.

\section{Matching arbitrary functions.}

4.1. Now we let $E$ be a quadratic extension of $F$, a nonarchimedean field of odd residual characteristic. Thus $E$ is an unramified or tamely ramified extension of $F$. We define the notion of "matching" for this more general situation. Recall that $Z$ denotes the center of $G$, a group isomorphic to $E^{\times}$, and that $Z^{\prime}$ denotes the center of $G^{\prime}$, a group isomorphic to $F^{\times}$. As before, we have an isomorphism

$$
H / Z \simeq G^{\prime} / Z^{\prime}
$$

unique within an inner automorphism, which allows us to transport a measure on $G^{\prime} / Z^{\prime}$ to $H / Z$. We define the function $f^{\prime}$ on $G^{\prime}$ as before. To complete our definition, we need to specify the function $f$ in terms of the function $f^{\prime}$. To that end we choose $\tau_{0}$ such that $E=F\left(\sqrt{\tau_{0}}\right)$; if $E$ is unramified, we take for $\tau_{0}$ a unit which is not a square, and if $E$ is (tamely) ramified, we take for $\tau_{0}$ a uniformizer of $F$. We let $\xi$ be the isomorphism

$$
\xi: H \rightarrow G^{\prime} Z
$$


defined by

$$
\xi(g)=\left(\begin{array}{cc}
\sqrt{\tau_{0}} & 0 \\
0 & 1
\end{array}\right) g\left(\begin{array}{cc}
{\sqrt{\tau_{0}}}^{-1} & 0 \\
0 & 1
\end{array}\right)
$$

We may view $f^{\prime}$ as a function on $G^{\prime} Z$ invariant under $Z$. Thus we may set

$$
f(g)=f^{\prime}(\xi(g))
$$

Let $U$ be the intersection $Z \cap G L\left(2, R_{E}\right)$. Thus $U$ is isomorphic to the group of units of $E$ and $K^{\prime} U$ is a maximal compact subgroup of $G^{\prime} Z$. We set $K_{H}=\xi^{-1}\left(K^{\prime} U\right)$. Hence $K_{H}$ is a maximal compact subgroup of $H$. Note that $f$ and $K_{H}$ do not depend on the choice of $\tau_{0}$, provided $\tau_{0}$ is as above. As before, we consider the integrals

$$
\begin{gathered}
\Omega(\Phi, \sigma, s)=\int f(h, s) \Phi\left[h^{-1} z \sigma h\right] \eta(\lambda(h)) d h d^{\times} z, \\
\Omega^{\prime}\left(\Phi^{\prime}, \sigma, s\right)=\int f^{\prime}\left(g^{\prime}, s\right) \Phi^{\prime}\left[g^{\prime-1} z \sigma g^{\prime}\right] d g^{\prime} \eta(z) d^{\times} z .
\end{gathered}
$$

As before, the integrals converge absolutely for $\mathfrak{R} s>0$ and $\sigma \in S_{0}$

$$
\sigma=\left(\begin{array}{cc}
\alpha & \beta_{1} \\
\beta_{2} & \alpha
\end{array}\right), \quad \beta_{1} \beta_{2} \neq 0 .
$$

Moreover, their interpretation as "Tate integrals" show that they continue analytically as meromorphic functions to the whole complex plane.

We shall say that a smooth function of compact support $\Phi$ on $S$ and a smooth function of compact support $\Phi^{\prime}$ on $G^{\prime}$ have matching orbital integrals if, for all $\sigma$ in $S_{0}$ of the above form, relation

$$
\left|\tau_{0}\right|^{-1 / 2} \Omega(\Phi, \sigma, s) L\left(s, 1_{F}\right)=\eta\left(\beta_{1}\right) \Omega^{\prime}\left(\Phi^{\prime}, \sigma, s\right) L(s, \eta)
$$

holds for all $s$ with $\Re s>0$. This definition is independent of the choice of $\tau_{0}$ and coincides with the definition of the previous section when $E$ is unramified.

As before, we will introduce the notations $\Omega(\Phi, \beta, s)$ and $\Omega^{\infty}(\Phi, \beta, s)$ and the corresponding notations for $\Omega^{\prime}$.

In this section, our goal is the following proposition:

Proposition 4.1. Given a smooth function of compact support $\Phi$ on $S$, there is a smooth function of compact support $\Phi^{\prime}$ on $G^{\prime}$ with matching orbital integrals. Conversely, given $\Phi^{\prime}$ there is $\Phi$ with matching orbital integrals.

To be definite, we prove the existence of $\Phi^{\prime}$ for a given $\Phi$. 
4.2. We first study the asympotic behavior of the integrals $\Omega(\Phi, \beta, s)$ when $\beta$ tends to 0. Explicitly,

$$
\Omega(\Phi, \beta, s)=\int \Phi\left[k^{-1}\left(\begin{array}{cc}
z(1-x) & z t^{-1}\left(\beta-x^{2}\right) \\
z t & z(1+x)
\end{array}\right) k\right] \eta(\lambda(k)) d k|t|^{s-1} \eta(t) d^{\times} t d x d^{\times} z .
$$

Here $x=u / \sqrt{\tau_{0}}$ and $d x=d u$.

We will denote by $I(\Phi, s)$ the integral corresponding to $\beta=0$. Explicitly,

$$
\begin{aligned}
I(\Phi, s) & =\int \Phi\left[h^{-1} z\left(\begin{array}{cc}
1 & 0 \\
1 & 1
\end{array}\right) h\right] f(h, s) \eta(\lambda(h)) d h \\
& =\int \Phi\left[k^{-1}\left(\begin{array}{cc}
z(1-x) & -z t^{-1} x^{2} \\
z t & z(1+x)
\end{array}\right) k\right] \eta(\lambda(k)) d k|t|^{s-1} \eta(t) d^{\times} t d x d^{\times} z .
\end{aligned}
$$

This integral is thus a unipotent orbital integral. It will be convenient to introduce the scalar part of the intertwining operator:

$$
m(s)=\int_{N_{H}} f(w n k, s) d n=\int_{N_{H}} f(w n, s) d n .
$$

Since $\lambda(w)=1$, we can change $h$ to $w h$ in the integral and then use the Iwasawa decomposition to obtain

$$
I(\Phi, s)=m(s) \int \Phi\left[k^{-1} z\left(\begin{array}{ll}
1 & t \\
0 & 1
\end{array}\right) k\right] \eta(\lambda(k)) d k|t|^{s} \eta(t) d^{\times} t d^{\times} z .
$$

In particular, this shows that the integral converges absolutely for $\mathfrak{R} s>0$.

We consider also the integral

$$
J(\Phi, s)=\int \Phi\left[h^{-1} z\left(\begin{array}{ll}
1 & 1 \\
0 & 1
\end{array}\right) h\right] \eta(\lambda(h)) f(h, s) d h d^{\times} z,
$$

the integral over the quotient $Z N_{H} \backslash H$. Explicitly,

$$
J(\Phi, s)=\int \Phi\left[k^{-1} z\left(\begin{array}{ll}
1 & t \\
0 & 1
\end{array}\right) k\right] \eta(\lambda(k)) d k d^{\times} z|t|^{1-s} \eta(t) d^{\times} t .
$$

This integral converges for $\Re s<1$.

It will be convenient to introduce the following function $\phi$ on $F$ :

$$
\phi(x)=\int \Phi\left[k^{-1} z\left(\begin{array}{ll}
1 & x \\
0 & 1
\end{array}\right) k\right] \eta(\lambda(k)) d k d^{\times} z
$$


Thus $\phi$ is a Schwartz-Bruhat function on $F$ such that

$$
\phi(u x)=\phi(x) \eta(u)
$$

for all $u \in F$ with $|u|=1$. It is easily seen that the function $\phi$ is an arbitrary Schwartz-Bruhat function with that property.

In terms of $\phi$,

$$
\begin{aligned}
& I(\Phi, s)=m(s) \int \phi(t)|t|^{s} \eta(t) d^{\times} t \\
& J(\Phi, s)=\int \phi(t)|t|^{1-s} \eta(t) d^{\times} t
\end{aligned}
$$

The first integral converges for $\mathfrak{R} s>0$ and the second for $\mathfrak{R}<<1$. These distributions determine the "Shalika germs" of the orbital integrals. Namely, let $A>0$ a constant and set for $\beta$ an integer

$$
C(\beta, s)=\int_{|x| \leqslant A}\left(\eta\left(\beta-x^{2}\right)\left|\beta-x^{2}\right|^{s-1}-\eta\left(-x^{2}\right)\left|x^{2}\right|^{s-1}\right) d x
$$

where

$$
x=\frac{u}{\sqrt{\tau_{0}}}, \quad u \in F, d x=d u .
$$

If we change the value of $A$, the integral does not change provided $|\beta|$ is small enough.

Proposition 4.2. Given $\Phi$, for $|\beta|$ sufficiently small and all $s$ with $0<\Re s<1$,

$$
\Omega(\Phi, \beta, s)=I(\Phi, s)+C(\beta, s) J(\Phi, s) .
$$

To prove the proposition, we use the following lemma:

LEMMA 4.3. Let $\chi$ be a fixed character of $F^{\times}$of module 1. Given a Schwartz-Bruhat function $\Psi$ on $F \times F$, there are two Schwartz-Bruhat functions $\Psi_{1}(x, u)$ and $\Psi_{2}(x, u)$ on $F$, which are Laurent polynomial in $q^{-u}$, such that

$$
\int \Psi\left(t, x t^{-1}\right) \chi(t)|t|^{u} d^{\times} t=L(u, \chi) \Psi_{1}(x, u)+L\left(-u, \chi^{-1}\right) \Psi_{2}(x, u) \chi(x)|x|^{u} .
$$

Moreover,

$$
\begin{aligned}
& \Psi_{1}(0, u)=\frac{\int \Psi(t, 0) \chi(t)|t|^{u} d^{\times} t}{L(u, \chi)} \\
& \Psi_{2}(0, u)=\frac{\int \Psi(0, t) \chi(t)^{-1}|t|^{-u} d^{\times} t}{L\left(-u, \chi^{-1}\right)} .
\end{aligned}
$$


To be more precise, each function $\Psi_{i}(x, u)$ is a finite sum of the form

$$
\sum_{j} \phi_{j}(x) q^{-u j}
$$

where the functions $\phi_{j}$ are Schwartz-Bruhat functions. The original integral converges for all $u$ provided $x \neq 0$, and the other integrals are meromorphic functions of $u$. We leave the proof of the lemma to the reader.

Next, we remark that, if $|\beta|<1$, then the corresponding matrix $\sigma$ has a determinant of absolute value one. It follows that, for a given $\Phi$, there is a compact set of $F^{\times}$with characteristic function $\mu$ such that

$$
\Omega(\Phi, \beta, s)=\int \Phi\left[h^{-1} z \sigma h\right] \eta(\lambda(h)) f(h, s) d h \mu(z) d^{\times} z .
$$

We apply our lemma (or rather a variant of the lemma with parameters) to the function $\Psi$ defined by

$$
\Psi\left(v, x_{1}, x_{2}\right)=\int \Phi\left[k^{-1}\left(\begin{array}{cc}
z(1+\bar{v}) & z x_{2} \\
z x_{1} & z(1+v)
\end{array}\right) k\right] \eta(\lambda(k)) d k \mu(z) d^{\times} z
$$

It is a Schwartz-Bruhat function on $E \times F \times F$. In terms of $\Psi$ (recall $x=u / \sqrt{\tau_{0}}$, $d x=d u$ ),

$$
\Omega(\Phi, \beta, s)=\int \Psi\left(x, t,\left(\beta-x^{2}\right) t^{-1}\right)|t|^{s-1} \eta(t) d^{\times} t d x .
$$

Thus we find

$$
\begin{aligned}
\Omega(\Phi, \beta, s)= & L(s-1, \eta) \int \Psi_{1}\left(x, \beta-x^{2}, s\right) d x \\
& +L(1-s, \eta) \int \Psi_{2}\left(x, \beta-x^{2}, s\right)\left|\beta-x^{2}\right|^{s-1} \eta\left(\beta-x^{2}\right) d x
\end{aligned}
$$

where the functions $\Psi_{i}$ are as in the lemma; here, they are Schwartz-Bruhat functions on $E \times F$ depending on $s$. For $|\beta|$ small enough we get,

$$
\begin{aligned}
\Omega(\Phi, \beta, s)= & L(s-1, \eta) \int \Psi_{1}\left(x,-x^{2}, s\right) d x \\
& +L(1-s, \eta) \int \Psi_{2}\left(x,-x^{2}, s\right)\left|\beta-x^{2}\right|^{s-1} \eta\left(\beta-x^{2}\right) d x
\end{aligned}
$$


Similarly, we get

$$
\begin{aligned}
I(\Phi, s)= & \int \Psi\left(x, t,-x^{2} t^{-1}\right)|t|^{s-1} \eta(t) d^{\times} t d x \\
= & L(s-1, \eta) \int \Psi_{1}\left(x,-x^{2}, s\right) d x \\
& +L(1-s, \eta) \int \Psi_{2}\left(x,-x^{2}, s\right)\left|x^{2}\right|^{s-1} \eta\left(-x^{2}\right) d x
\end{aligned}
$$

On the other hand,

$$
J(\Phi, s)=\int \Psi(0,0, t)|t|^{1-s} \eta(t) d^{\times} t .
$$

Thus, by the lemma

$$
J(\Phi, s)=L(1-s, \eta) \Psi_{2}(0,0, s)
$$

To obtain our assertion we write our orbital integral in the form

$$
\begin{aligned}
& L(s-1, \eta) \int \Psi_{1}\left(x,-x^{2}, s\right) d x+L(1-s, \eta) \int \Psi_{2}\left(x,-x^{2}, s\right)\left|x^{2}\right|^{s-1} \eta\left(-x^{2}\right) d x \\
& \quad+L(1-s, \eta) \int \Psi_{2}\left(x,-x^{2}, s\right)\left(\left|\beta-x^{2}\right|^{s-1} \eta\left(\beta-x^{2}\right)-\left|x^{2}\right|^{s-1} \eta\left(-x^{2}\right)\right) d x
\end{aligned}
$$

The sum of the two first terms is $I(\Phi, s)$. In the third term the bracket expression vanishes if $\left|x^{2}\right|$ is large in comparison with $\beta$. Thus, for $\beta$ small enough, we may replace $\Psi_{2}\left(x,-x^{2}, s\right)$ by $\Psi_{2}(0,0, s)$ in the third term. We get then

$$
\Omega(\Phi, \beta, s)=I(\Phi, s)+C(\beta, s) J(\Phi, s) .
$$

This proves the proposition.

4.3. We consider similarly the integrals $\Omega^{\prime}$. Recall the integral

$$
\Omega^{\prime}\left(\Phi^{\prime}, \beta, s\right)=\int \Phi^{\prime}\left[g^{-1} z \sigma g\right] f(g, s) d g \eta(z) d^{\times} z
$$

where

$$
\sigma=\left(\begin{array}{ll}
1 & \beta \\
1 & 1
\end{array}\right)
$$


Explicitly,

$$
\Omega^{\prime}\left(\Phi^{\prime}, \beta, s\right)=\int \Phi^{\prime}\left[k^{-1}\left(\begin{array}{cc}
z(1-x) & z t^{-1}\left(\beta-x^{2}\right) \\
z t & z(1+x)
\end{array}\right) k\right] d k|t|^{s-1} d^{\times} t d x \eta(z) d^{\times} z .
$$

As before, we consider the orbital integral corresponding to $\beta=0$ :

$$
I^{\prime}\left(\Phi^{\prime}, s\right)=\int \Phi^{\prime}\left[k^{-1}\left(\begin{array}{cc}
z(1-x) & -z t^{-1} x^{2} \\
z t & z(1+x)
\end{array}\right) k\right] d k|t|^{s-1} d^{\times} t d x \eta(z) d^{\times} z .
$$

We denote by $N^{\prime}$ the intersection of $G^{\prime}$ and $N$ and denote by $m^{\prime}(s)$ the scalar part of the intertwining operator

$$
m^{\prime}(s)=\int_{N^{\prime}} f^{\prime}(w n k, s) d n=\int_{N^{\prime}} f^{\prime}(w n, s) d n
$$

We have then

$$
I^{\prime}\left(\Phi^{\prime}, s\right)=m^{\prime}(s) \int \Phi^{\prime}\left[k^{-1} z\left(\begin{array}{ll}
1 & t \\
0 & 1
\end{array}\right) k\right] d k|t|^{s} d^{\times} \operatorname{t\eta }(z) d^{\times} z
$$

Similarly, we define

$$
J^{\prime}\left(\Phi^{\prime}, s\right)=\int \Phi^{\prime}\left[g^{\prime-1} z\left(\begin{array}{ll}
1 & 1 \\
0 & 1
\end{array}\right) g^{\prime}\right] f^{\prime}\left(g^{\prime}, s\right) d g^{\prime} \eta(z) d^{\times} z
$$

the integral over $Z^{\prime} N^{\prime} \backslash G^{\prime}$. Explicitly,

$$
J^{\prime}\left(\Phi^{\prime}, s\right)=\int \Phi^{\prime}\left[k^{-1} z\left(\begin{array}{ll}
1 & t \\
0 & 1
\end{array}\right) k\right] d k \eta(z) d^{\times} z|t|^{1-s} d^{\times} t
$$

As before, it will be convenient to introduce the following function $\phi^{\prime}$ on $F$ :

$$
\phi^{\prime}(x)=\int \Phi^{\prime}\left[k^{-1} z\left(\begin{array}{ll}
1 & x \\
0 & 1
\end{array}\right) k\right] d k \eta(z) d^{\times} z .
$$

Thus $\phi^{\prime}$ is a Schwartz-Bruhat function on $F$ such that

$$
\phi^{\prime}(u x)=\phi^{\prime}(x)
$$


for all $u \in F$ with $|u|=1$. As before, the function $\phi^{\prime}$ is an arbitrary Schwartz-Bruhat function with that property. In terms of $\phi^{\prime}$,

$$
\begin{aligned}
& I^{\prime}\left(\Phi^{\prime}, s\right)=m^{\prime}(s) \int \phi^{\prime}(t)|t|^{s} d^{\times} t \\
& J^{\prime}\left(\Phi^{\prime}, s\right)=\int \phi^{\prime}(t)|t|^{1-s} d^{\times} t .
\end{aligned}
$$

We define

$$
C^{\prime}(\beta, s)=\int_{|x| \leqslant A}\left(\left|\beta-x^{2}\right|^{s-1}-\left|x^{2}\right|^{s-1}\right) d x
$$

As before, this does not depend on the value on $A$, provided $|\beta|$ is small enough and we have the following proposition:

Proposition 4.4. Given $\Phi^{\prime}$, for $|\beta|$ sufficiently small and all $s$ with $\mathfrak{R} s>0$,

$$
\Omega^{\prime}\left(\Phi^{\prime}, \beta, s\right)=I^{\prime}\left(\Phi^{\prime}, s\right)+C^{\prime}(\beta, s) J^{\prime}\left(\Phi^{\prime}, s\right)
$$

The proof is the same as before.

4.4. The first step in matching the orbital integrals is the following lemma:

LEMMA 4.5. Given $\Phi$, there is $\Phi^{\prime}$ such that for all s and all $\beta$ sufficiently small,

$$
\left|\tau_{0}\right|^{-1 / 2} \Omega(\Phi, \beta, s) L\left(s, 1_{F}\right)=\Omega^{\prime}\left(\Phi^{\prime}, \beta, s\right) L(s, \eta)
$$

Proof. It suffices to show that given $\Phi$ there is $\Phi^{\prime}$ with

$$
\begin{gathered}
\left|\tau_{0}\right|^{-1 / 2} I(\Phi, s) L\left(s, 1_{F}\right)=I^{\prime}\left(\Phi^{\prime}, s\right) L(s, \eta) \\
\left|\tau_{0}\right|^{-1 / 2} C(\beta, s) J(\Phi, s) L\left(s, 1_{F}\right)=C^{\prime}(\beta, s) J^{\prime}\left(\Phi^{\prime}, s\right) L(s, \eta) .
\end{gathered}
$$

Now it is easily verified that

$$
m(s)=m^{\prime}(s)\left|\tau_{0}\right|^{s}
$$

The relation (23) reads

$$
\left|\tau_{0}\right|^{s-1 / 2} \int \phi(x)|x|^{s} \eta(x) d^{\times} x / L(s, \eta)=\int \phi^{\prime}(x)|x|^{s} d^{\times} x / L\left(s, 1_{F}\right) .
$$


The left-hand side is a Laurent polynomial in $q^{-s}$. Since $\phi^{\prime}$ is an arbitrary SchwartzBruhat function such that

$$
\phi^{\prime}(x u)=\phi^{\prime}(x)
$$

for $|u|=1$, we see that the right-hand side is an arbitrary Laurent polynomial and so we can choose $\Phi^{\prime}$ in such a way that this relation and thus (23) is satisfied. Then the relation (24) will be a consequence of the following identity that we state as a lemma:

LEMMA 4.6. With the above notations,

$$
\left|\tau_{0}\right|^{s-1} \frac{L\left(s, 1_{F}\right) C(\beta, s)}{L\left(1-s, 1_{F}\right)}=\frac{L(s, \eta) C^{\prime}(\beta, s)}{L(1-s, \eta)},
$$

provided $|\beta|$ is small enough.

As before, we set $X=q^{-s}$ and we write $C(\beta, X)$ and $C^{\prime}(\beta, X)$ for $C$ and $C^{\prime}$. The function $C^{\prime}$ is easily computed: we let $\tau$ be a unit of $F$ which is not a square, $\varpi$ a uniformizer of $F$, and $y$ be an integer of $F$; then

$$
C^{\prime}(\beta, X)= \begin{cases}-q^{-1}|y|^{2 s-1} \frac{(X q-1)^{2}(1+X)}{\left(1-X^{2} q\right)(1-X)} & \text { if } \beta=y^{2} \\ -q^{-1}|y|^{2 s-1} \frac{\left(X^{2} q^{2}-1\right)}{1-X^{2} q} & \text { if } \beta=y^{2} \tau \\ |y|^{2 s-1} \frac{X(1-X q)(1+X)}{1-X^{2} q} & \text { if } \beta=y^{2} \varpi .\end{cases}
$$

In particular, we have the functional equation

$$
\frac{1-X^{-1} q^{-1}}{1-X} C^{\prime}\left(\frac{\beta}{\tau},-X\right)=\frac{1+X^{-1} q^{-1}}{1+X} C^{\prime}(\beta, X) .
$$

Now suppose the extension unramified. Then $\tau_{0}$ is a unit and

$$
\eta\left(\tau_{0}\right)=\eta\left(-\tau_{0}\right)=1
$$

We have then

$$
\begin{aligned}
C(\beta, s) & =\int\left[\eta\left(\beta-\frac{u^{2}}{\tau_{0}}\right)\left|\beta-\frac{u^{2}}{\tau_{0}}\right|^{s-1}-\left|\frac{u^{2}}{\tau_{0}}\right|^{s-1}\right] d u \\
& =C^{\prime}\left(\beta \tau_{0},-X\right) .
\end{aligned}
$$


Thus

$$
\begin{aligned}
\frac{L(s, 1)}{L(1-s, 1)} C(\beta, s) & =\frac{1-X^{-1} q^{-1}}{1-X} C^{\prime}\left(\beta \tau_{0},-X\right) \\
& =\frac{1+X^{-1} q^{-1}}{1+X} C^{\prime}(\beta, X) \\
& =\frac{L(s, \eta)}{L(1-s, \eta)} C^{\prime}(\beta, s)
\end{aligned}
$$

by the functional equation (26). This proves the lemma in this case.

Now suppose the extension is ramified. Then $\tau_{0}$ is a uniformizer of $F$, and the relation to be proved reads

$$
q X \frac{1-X^{-1} q^{-1}}{1-X} C(\beta, s)=C^{\prime}(\beta, s)
$$

We first prove this relation when $\beta=y^{2}$ for some integer $y$. Then in the integral for $C$, both $\beta-x^{2}$ and $-x^{2}$ are norms. Thus we get

$$
\begin{aligned}
C\left(y^{2}, s\right) & =\int\left(\left|y^{2}-\frac{u^{2}}{\tau_{0}}\right|^{s-1}-\left|\frac{u^{2}}{\tau_{0}}\right|^{s-1}\right) d u \\
& =X^{-1} q^{-1} \int\left(\left|\tau_{0} y^{2}-u^{2}\right|^{s-1}-\left|u^{2}\right|^{s-1}\right) d u \\
& =X^{-1} q^{-1} C^{\prime}\left(y^{2} \tau_{0}, X\right) \\
& =X^{-1} q^{-1}|y|^{2 s-1} \frac{X(1-X q)(1+X)}{1-X^{2} q} .
\end{aligned}
$$

On the other hand,

$$
C^{\prime}\left(y^{2}, s\right)=-q^{-1}|y|^{2 s-1} \frac{(X q-1)^{2}(1+X)}{\left(1-X^{2} q\right)(1-X)}
$$

so that the relation (27) is easily verified.

For the remaining cases fresh computations are needed:

LEMMA 4.7. Suppose the extension is ramified so that $\tau_{0}$ is a uniformizer. Suppose also $\beta=y^{2} \tau_{1}$ where $\tau_{1}$ is not a square and $y$ is an integer. If $\tau_{1}$ is a unit, then

$$
C(\beta, s)=|y|^{2 s-1} \frac{q^{-1}(1+X q)(X-1)}{1-X^{2} q} .
$$


If $\tau_{1}$ is a uniformizer, then

$$
C(\beta, s)=|y|^{2 s-1} \frac{-X\left(1-X^{2}\right)}{1-X^{2} q} .
$$

The relation (27) follows easily by comparing with the corresponding formula for $C^{\prime}$. It remains to establish the lemma.

First suppose $\tau_{1}$ is a unit. Then $\eta\left(\tau_{1}\right)=-1$. After changing $u$ to $u \tau_{0}$, we get

$$
C\left(y^{2} \tau_{1}, s\right)=q^{-1} \int_{|u| \leqslant 1}\left(\eta\left(y^{2} \tau_{1}-u^{2} \tau_{0}\right)\left|y^{2} \tau_{1}-u^{2} \tau_{0}\right|^{s-1}-\left|u^{2} \tau_{0}\right|^{s-1} \eta\left(-u^{2} \tau_{0}\right)\right) d u .
$$

For $|u|>|y|$ the integrand is zero. Thus the integral simplifies to

$$
q^{-1} \int_{|u| \leqslant|y|}\left(\eta\left(\tau_{1}\right)\left|y^{2} \tau_{1}-u^{2} \tau_{0}\right|^{s-1}-\left|u^{2} \tau_{0}\right|^{s-1}\right) d u .
$$

Changing $u$ to $y u$, we get

$$
q^{-1}|y|^{2 s-1} \int_{|u| \leqslant 1}\left(-\left|\tau_{1}-u^{2} \tau_{0}\right|^{s-1}-\left|u^{2} \tau_{0}\right|^{s-1}\right) d u
$$

which simplifies further to

$$
q^{-1}|y|^{2 s-1}\left[-1-\int_{|u| \leqslant 1} X q\left|u^{2}\right|^{s-1} d u\right]
$$

This is easily evaluated to give the answer.

Now assume $\tau_{1}$ is a uniformizer. Recall $\tau_{0}$ is also assumed to be a uniformizer. We have again

$$
C(\beta, s)=q^{-1} \int_{|u| \leqslant 1}\left(\eta\left(y^{2} \tau_{1}-\tau_{0} u^{2}\right)\left|\tau_{1} y^{2}-\tau_{0} u^{2}\right|^{s-1}-\left|\tau_{0} u^{2}\right|^{s-1}\right) d u .
$$

As before, the integrand is zero for $|u|>|y|$. After changing $u$ to $u y$, we find for the integral

$$
q^{-1}|y|^{2 s-1} \int_{|u| \leqslant 1}\left(\eta\left(\tau_{1}-\tau_{0} u^{2}\right)\left|\tau_{1}-\tau_{0} u^{2}\right|^{s-1}-\left|\tau_{0} u^{2}\right|^{s-1}\right) d u .
$$

We write the integral of this difference as a difference of two integrals, the first of which we decompose as the sum of the contributions of the sets

$$
|u|=1, \quad|u|<1
$$


In this way we obtain

$$
q^{-1}|y|^{2 s-1}\left[\int_{|u|=1} \eta\left(\tau_{1}-\tau_{0} u^{2}\right)\left|\tau_{1}-\tau_{0} u^{2}\right|^{s-1} d u+X \eta\left(\tau_{1}\right)-\frac{X q\left(1-q^{-1}\right)}{1-X^{2} q}\right] .
$$

The rest of the computation depends on whether or not $\tau_{1} / \tau_{0}$ is a square. First assume it is not. Then

$$
\left|\tau_{1}-\tau_{0} u^{2}\right|^{s-1}=X q
$$

and

$$
\eta\left(\tau_{1}\right)=\eta\left(\tau_{1} / \tau_{0}\right) \eta\left(-\tau_{0}\right) \eta(-1)=-\eta(-1)
$$

Thus the above expression becomes

$$
q^{-1}|y|^{2 s-1}\left[-\eta(-1) X q \int_{|u|=1} \eta\left(1-\varepsilon u^{2}\right) d u-\eta(-1) X-\frac{X q\left(1-q^{-1}\right)}{1-X^{2} q}\right],
$$

where $\varepsilon=\tau_{0} / \tau_{1}$. Thus $\varepsilon$ is a unit and not a square. Let $k$ be the residual field of $F$; thus $k$ has $q$ elements, and we may view $\eta$ as the unique nontrivial quadratic character of $k^{\times}$. The integral which remains to be computed can be evaluated as a sum over $k^{\times}$:

$$
q \int_{|u|=1} \eta\left(1-u^{2} \varepsilon\right) d u=\sum_{x \in k^{\star}} \eta\left(1-x^{2} \varepsilon\right)
$$

Thus the total expression to be evaluated is

$$
q^{-1}|y|^{2 s-1}\left[-\eta(-1) X\left(1+\sum \eta\left(1-\varepsilon x^{2}\right)\right)-\frac{X q\left(1-q^{-1}\right)}{1-X^{2} q}\right] .
$$

Since $\varepsilon$ is not a square, $1-x^{2} \varepsilon$ is not zero and the sum over $x$ is also equal to

$$
2 \#\left\{x \neq 0 \mid 1-x^{2} \varepsilon \text { is a square }\right\}-(q-1)
$$

or, what amounts to the same,

$$
\#\left\{(x, y) \mid x^{2} \varepsilon+y^{2}=1\right\}-2-(q-1)
$$

If -1 is a square, then $\eta(-1)=1$ and the quadratic form $x^{2} \varepsilon+y^{2}$ is equivalent to the norm form for the quadratic extension of $k$. Thus the above number of pairs is $q+1$, and the sum over $x$ is zero. The first term in the total expression is then $-X$. 
If -1 is not a square, then $\eta(-1)=-1$ and the quadratic form $x^{2} \varepsilon+y^{2}$ is equivalent to the form $x y$ so that the number of pairs is now $q-1$. The sum over $x$ is then equal to -2 , and the first term in the total expression is still equal to $-X$. Thus the total expression is

$$
q^{-1}|y|^{2 s-1}\left[-X-\frac{X q\left(1-q^{-1}\right)}{1-X^{2} q}\right]
$$

This simplifies to the required expression for Lemma 4.7.

Now assume $\tau_{0} / \tau_{1}$ is a square. We may assume $\tau_{1}=\tau_{0}$ as well. Then $\eta\left(\tau_{0}\right)=$ $\eta(-1)$. We find

$$
q^{-1}|y|^{2 s-1}\left[\eta(-1) X q \int_{|u|=1} \eta\left(1-u^{2}\right)\left|1-u^{2}\right|^{s-1} d u+X \eta(-1)-\frac{X q\left(1-q^{-1}\right)}{1-X^{2} q}\right] .
$$

Because the character $\eta$ is ramified, the contribution of the sets $|u+1|<1$ and $|u-1|<1$ vanishes. Thus the integral can be replaced by an integral over the set

$$
|u|=|u+1|=|u-1|=1
$$

or by a sum over $k^{\times}$

$$
q \int \eta\left(1-u^{2}\right)\left|1-u^{2}\right|^{s-1} d u=q \int \eta\left(1-u^{2}\right) d u=\sum_{x \neq 0,1-x^{2} \neq 0} \eta\left(1-x^{2}\right) .
$$

In this way the total expression is equal to

$$
|y|^{2 s-1}\left[\eta(-1) X\left(1+\sum_{x \neq 0,1-x^{2} \neq 0} \eta\left(1-x^{2}\right)\right)-\frac{X q\left(1-q^{-1}\right)}{1-X^{2} q}\right] .
$$

Now

$$
\begin{aligned}
\sum_{x \neq 0,1-x^{2} \neq 0} \eta\left(1-x^{2}\right)= & 2 \#\left\{x \neq 0 \mid 1-x^{2} \neq 0,1-x^{2} \text { is a square }\right\} \\
& -\#\left\{x \neq 0 \mid 1-x^{2} \neq 0\right\} \\
= & \#\left\{(x, y) \mid x^{2}+y^{2}=1\right\}-q-1
\end{aligned}
$$

If -1 is a square, we have $\eta(-1)=1$; the form $x^{2}+y^{2}$ is then equivalent to $x y$ and the number of pairs is $q-1$. The sum over $x$ is thus equal to -2 , and the first term in the total expression is $-X$. If -1 is not a square, then $\eta(-1)=-1$; the form is equivalent to the norm form and the number of pairs is $q+1$. Thus the sum is 0 , and the first term in the total expression is again $-X$. Thus we find the same result as before and we are done. This completes the proof of Lemmas 4.7 and 4.5. 
4.5. To complete the proof of Proposition 4.1, it will suffice to show the following statement. We choose a set of representatives $\{\tau\}$ for the cosets $F^{\times} / F^{\times 2}$.

LEMMA 4.8. Given $\tau$ and

$$
\sigma_{0}=\left(\begin{array}{cc}
\alpha_{0} & \beta_{0} \tau \\
\beta_{0} & \alpha_{0}
\end{array}\right)
$$

with $\beta_{0} \neq 0$, let $T_{0}$ be the torus which centralizes $\sigma_{0}$ in $G L(2, E)$. Its intersection with $H$ is a torus $T$ in $H$, and its intersection with $G^{\prime}$ a torus $T^{\prime}$ in $G^{\prime}$. Let $U$ be a compact open set of $T^{\prime}$ containing $\sigma_{0}$. Assume furthermore that $U$ does not contain a pair of the form

$$
\left(\begin{array}{cc}
\alpha & \beta \tau \\
\beta & \alpha
\end{array}\right),\left(\begin{array}{cc}
\alpha & -\beta \tau \\
-\beta & \alpha
\end{array}\right)
$$

or an element with $\beta \neq 0$. Then there is $\Phi^{\prime}$ such that

$$
\eta(\beta) \Omega(\Phi, \sigma, s) L(s, 1)=\Omega^{\prime}\left(\Phi^{\prime}, \sigma, s\right) L(s, \eta)
$$

for $\sigma$ in $U$, and

$$
\Omega^{\prime}\left(\Phi^{\prime}, \sigma, s\right)=0
$$

if $\sigma$ is not conjugate to an element of $F^{\times} U$.

Proof. We can write $\Omega$ in the form

$$
\Omega(\Phi, \sigma, s)=\int_{T \backslash \boldsymbol{H}} \Phi\left(h^{-1} z \sigma h\right) \eta(\lambda(h)) d h \int_{\boldsymbol{Z} \backslash \boldsymbol{T}} f(t h, s) d t d^{\times} z .
$$

The relations

$$
\sigma \in U, h^{-1} \sigma z h \in \operatorname{Support} \Phi
$$

imply that $z$ is in a compact set of $F^{\times}$and $h$ in a fixed compact set of $T \backslash H$. Thus, for $\sigma \in U$ we can write

$$
\Omega(\Phi, \sigma, s)=\int \phi(h, \sigma) \eta(\lambda(h)) f(h, s) d h
$$

where $\phi$ is a smooth function on $T \backslash H \times F^{\times} U$, such that

$$
\phi(h, z u)=\phi(h, u)
$$


and whose support has a compact projection on the first factor. Similarly, we can choose a $\Phi^{\prime}$ whose support is compact and contained in the set of conjugates of $U$ in $G^{\prime}$. Then $\Omega^{\prime}$ vanishes for $\sigma$ not conjugate into $U F^{\times}$. For $\sigma$ in $U$, it can be written

$$
\Omega^{\prime}\left(\Phi^{\prime}, \sigma, s\right)=\int \phi^{\prime}\left(g^{\prime}, \sigma\right) f\left(g^{\prime}, s\right) d g^{\prime}
$$

where $\phi^{\prime}$ is an arbitrary smooth function on $T^{\prime} \backslash G^{\prime} \times U F^{\times}$such that

$$
\phi^{\prime}(h, z u)=\eta(z) \phi^{\prime}(h, u)
$$

and whose support has a compact projection on the first factor. Thus it will suffice to show that, given $\phi$, we can find $\phi^{\prime}$ such that

$$
L(s, 1) \eta(\beta) \int \phi(h, \sigma) \eta(\lambda(h)) f(h, s) d h=L(s, \eta) \int \phi^{\prime}\left(g^{\prime}, \sigma\right) f^{\prime}\left(g^{\prime}, s\right) d h
$$

for $\sigma \in U$. In other words, it will suffice to show that, given $h \in H$, there are $g_{i} \in G^{\prime}$ and $c_{i} \in \mathbb{C}$ such that

$$
L(s, 1) \int f(t h, s) \eta(\lambda(t h)) d t=L(s, \eta) \sum c_{i} \int f^{\prime}\left(t^{\prime} g_{i}, s\right) d t^{\prime}
$$

We can use the isomorphism between $H / Z$ and $G^{\prime} / Z^{\prime}$ to reduce this assertion to a question on $G^{\prime}: T^{\prime}$ is as before but $T$ is now a torus of $G^{\prime}$. We can take it to be the torus which centralizes the element

$$
\left(\begin{array}{cc}
1 & \tau \tau_{0} \\
1 & 1
\end{array}\right)
$$

in $G^{\prime}$. We can take $f$ to be $f^{\prime}$ and replace $\lambda$ by det. Furthermore, if $\Phi_{0}$ is the characteristic function of $R_{F} \times R_{F}$ in $F^{2}$, we can take

$$
f^{\prime}(g, s)=\int \Phi_{0}[(0, u) g]|u|^{2 s} d^{\times} u|\operatorname{det} g|^{s} .
$$

The above identity reads

$$
\int_{T / Z^{\prime}} f^{\prime}(t h, s) \eta(\operatorname{det}(t h)) d t / L(s, \eta)=\sum c_{i} \int_{T^{\prime} / Z^{\prime}} f\left(t^{\prime} g_{i}, s\right) d t^{\prime} / L(s, 1) .
$$

We can view $T^{\prime}$ as the multiplicative group of an algebra $L^{\prime}$ isomorphic to $F \times F$ if $\tau$ is a square and to $F(\sqrt{\tau})$ if not. In particular (see [JZ]),

$$
\int_{T^{\prime} / Z^{\prime}} f^{\prime}\left(t^{\prime} g, s\right) d t^{\prime}=\int_{T^{\prime}} \Phi_{0}\left[(0,1) t^{\prime} g\right]|\operatorname{det} t g|^{s} d^{\times} t^{\prime}
$$


is a Tate integral for $L^{\prime}$ and the trivial character. The corresponding $L$-function is $L(s, 1) L\left(s, \eta_{\tau}\right)$.

A similar interpretation is available for $T$ as multiplicative group of an algebra $L$ isomorphic to $F \times F$ if $\tau \tau_{0}$ is a square and to $F\left(\sqrt{\tau \tau_{0}}\right)$ if not. Similarly,

$$
\int_{T / Z^{\prime}} f^{\prime}(t h, s) \eta(\operatorname{det}(t h)) d t=\int_{T} \Phi_{0}[(0,1) t h]|\operatorname{det} t h|^{s} \eta(\operatorname{det} t h) d^{\times} t
$$

is a Tate integral for the character $\eta \circ \operatorname{det}$ of $L$. The corresponding $L$-function is $L(s, \eta) L\left(s, \eta_{\tau}\right)$.

The proposition will then follow from the next lemma:

LEMMA 4.9. Let $\Phi_{0}$ be the characteristic function of $R_{F}^{2}$ in $F^{2}$. Assume $\tau$ is a unit or a uniformizer. Set $X=q^{-s}$ and

$$
P(X)=\int_{T^{\prime}} \Phi_{0}\left[(0,1) t^{\prime} g\right]\left|\operatorname{det} t^{\prime} g\right|^{s} d t^{\prime} / L(s, 1) L\left(s, \eta_{\tau}\right) .
$$

Then

$$
P(X)=P\left(X^{-1} q^{-1}\right)
$$

and any Laurent polynomial satisfying this identity can be written as as sum of expressions of the above type. The same assertion is true for

$$
Q(X)=\int_{T} \Phi_{0}[(0,1) t g] \eta(\operatorname{det} t g)|\operatorname{det} t g|^{s} d t / L(s, \eta) L\left(s, \eta_{\tau}\right)
$$

4.6. We now prove the lemma. This requires some preparation. It will be convenient to say that a Laurent polynomial is symmetric if it satisfies (28). Let $\psi_{0}$ be an additive character of $F$ whose conductor is $R_{F}$. We define the Fourier transform of a Schwartz-Bruhat function $\phi$ on $F$ to be

$$
\hat{\phi}(x)=\int \phi(y) \psi_{0}(x y) d y,
$$

the measure being self-dual. We define the symplectic Fourier transform of a Schwartz-Bruhat function $\Phi$ on $F^{2}$ by

$$
\widehat{\Phi}(x, y)=\iint \Phi(u, v) \psi_{0}(u y-v x) d u d v
$$

It follows that for $g \in S L(2, F)$ (or for $|\operatorname{det} g|=1$ ) the function

$$
(x, y) \mapsto \Phi_{0}[(x, y) g]
$$


is its own symplectic Fourier transform. Conversely, we have the following statement.

LEMMA 4.10. Let $V$ be the space of Schwartz-Bruhat functions $\Phi$ on $F^{2}$ such that

$$
\widehat{\Phi}=\Phi, \quad \Phi[u(x, y)]=\Phi[(x, y)] \quad \text { for }|u|=1 .
$$

Then $V$ is spanned by the functions of the form

$$
(x, y) \mapsto \Phi_{0}[(x, y) g]
$$

with $\operatorname{det} g=1$.

This is a standard result ([Ho]). Similarly, we have the following lemma.

LEMMA 4.11. Let $V_{1}$ be the space of Schwartz-Bruhat functions $\Phi$ on $F^{2}$ such that

$$
\hat{\Phi}(x, y)=q \Phi\left(\varpi^{-1} x, \varpi^{-1} y\right), \quad \Phi[u(x, y)]=\Phi[(x, y)] \quad \text { for }|u|=1 .
$$

Then $V_{1}$ is spanned by the functions of the form

$$
(x, y) \mapsto \Phi_{0}[(x \varpi, y) g]
$$

with $\operatorname{det} g=1$.

4.7. Now we prove the assertions relative to the polynomial $P$ in Lemma 4.9. We can assume that $\tau$ is either a unit or a uniformizer.

First suppose $\tau$ is a uniformizer. Then the image of $T^{\prime}$ under det is the norm group for a ramified extension. Its product by the group of units is the whole multiplicative group of $F$. Thus we can take $g$ in $S L(2, F)$ without restricting the generality. By Lemma 4.10 we can write

$$
P(X)=\int_{T^{\prime}} \Phi\left[(0,1) t^{\prime}\right]\left|\operatorname{det} t^{\prime}\right|^{s} d t^{\prime} / L(s, 1) L\left(s, \eta_{\tau}\right),
$$

where $\Phi$ is an arbitrary element of $V$. We have to show that $P$ is an arbitrary symmetric polynomial. Recall the algebra $L^{\prime}$ of matrices of the form

$$
\left(\begin{array}{cc}
a & b \tau \\
b & a
\end{array}\right)
$$

Thus $L^{\prime} \simeq F(\sqrt{\tau})$ and

$$
L\left(s, 1_{L^{\prime}}\right)=L(s, 1) L\left(s, \eta_{\tau}\right)
$$


We identify $\Phi$ to the function $\Psi$ on $L^{\prime}$ defined by

$$
\Psi(a+b \sqrt{\tau})=\Phi(b, a)
$$

We define an additive character $\psi$ on $L^{\prime}$ by

$$
\psi(z)=\psi_{0}\left(\operatorname{Tr}\left(\frac{z}{2 \sqrt{\tau}}\right)\right) .
$$

The corresponding Fourier transform is

$$
\hat{\Psi}(z)=\int \Psi(u) \psi(u z) d u,
$$

the measure being self-dual. Note that $\hat{\Phi}$ is identified to $\hat{\Psi}(\bar{z})$. We now have

$$
P(X)=\int_{L^{\prime}} \Psi(z)|z|^{s} d^{\times} z / L\left(s, 1_{L^{\prime}}\right)
$$

where $\Psi$ is in the space $V_{L^{\prime}}$ of functions $\Psi$ such that

$$
\Psi(z)=\hat{\Psi}(\bar{z}), \quad \Psi(u z)=\Psi(z), \quad u \in R_{F}^{\times} .
$$

Now $\Phi_{0}$ is its own Fourier transform. The corresponding function on $L^{\prime}$ is the characteristic function of the ring $R_{L^{\prime}}$ of integers in $L^{\prime}$. It follows that the Tate functional equation takes the form

$$
\int_{L^{\prime}} \Psi(z)|z|^{s} d^{\times} z / L\left(s, 1_{L^{\prime}}\right)=\int_{L^{\prime}} \hat{\Psi}(z)|z|^{1-s} d^{\times} z / L\left(1-s, 1_{L^{\prime}}\right) .
$$

Since $L^{\prime}$ is a ramified quadratic extension, the cardinality of its residual field is $q$. For $\Psi \in V_{L^{\prime}}$, the left-hand side is thus a symmetric polynomial in $X=q^{-s}$. Moreover, it is easily checked that it is in fact an arbitrary symmetric polynomial. This proves our assertion in this case.

Now assume that $\tau$ is a unit but not a square. Then the image of $T^{\prime}$ under det is the set of elements with even valuation. This time we may assume $|\operatorname{det} g|$ is 1 or $q^{-1}$. This leads us to consider the polynomials

$$
P(X)=\int_{T^{\prime}} \Phi\left[(0,1) t^{\prime}\right]\left|\operatorname{det} t^{\prime}\right|^{s} d t^{\prime} / L(s, 1) L\left(s, \eta_{\tau}\right),
$$

where $\Phi$ is an arbitrary element of $V$, and

$$
P_{1}(X)=X \int_{T^{\prime}} \Phi\left[(0,1) t^{\prime}\right]\left|\operatorname{det} t^{\prime}\right|^{s} d t^{\prime} / L(s, 1) L\left(s, \eta_{\tau}\right),
$$


where $\Phi_{1}$ is an arbitrary element of $V_{1}$. We have to show that they are symmetric and that they span, when the functions $\Phi$ vary, the space of symmetric polynomials. As before, we introduce the algebra $L^{\prime}$ and the character $\psi$, and the Tate functional equation takes the form (29). We can write

$$
P(X)=\int_{L^{\prime}} \Psi(z)|z|^{s} d^{\times} z / L\left(s, 1_{L^{\prime}}\right),
$$

where $\Psi$ is in the space $V_{L^{\prime}}$ corresponding to $V$. Since the residual field of $L^{\prime}$ has $q^{2}$ elements, $P(X)$ is an arbitrary symmetric polynomial in $X^{2}$. Similarly, the space $V_{1 L^{\prime}}$ of the functions $\Psi$ corresponding to the functions $\Phi \in V_{1}$ consists of all functions $\Psi$ such that

$$
\hat{\Psi}(\bar{z})=q \Psi\left(\varpi^{-1} z\right), \quad \Psi(u z)=\Psi(z) \quad \text { for } u \in R_{F}^{\times} .
$$

We then have

$$
P_{1}(X)=X \int_{L^{\prime}} \Psi(z)|z|^{s} d^{\times} z / L\left(s, 1_{L^{\prime}}\right),
$$

with $\Psi \in V_{1 L^{\prime}}$. Applying the Tate functional equation, we find that $P_{1}$ is a symmetric polynomial containing odd-degree monomials only. It is easily seen that $P_{1}$ is an arbitrary polynomial with these properties. Our assertion is proved in the case at hand.

We pass to the case where $\tau$ is a unit and a square. After a change of variables, we can take $\tau=1$. Moreover, without a loss of generality, we can take $g \in S L(2, F)$. Thus we can take

$$
P(X)=\int_{T^{\prime}} \Phi\left[(0,1) t^{\prime}\right]\left|\operatorname{det} t^{\prime}\right|^{s} d t^{\prime} / L\left(s, 1_{F}\right) L\left(s, 1_{F}\right),
$$

where $\Psi$ is in $V$. We have to show this is an arbitrary symmetric polynomial. The algebra $L^{\prime}$ is isomorphic to the sum of two copies of $F$. Correspondingly, we identify $\Phi$ to the function $\Psi$ on $L^{\prime}$ defined by

$$
\Psi(u, v)=\Phi(b, a)
$$

where

$$
u=a+b, \quad v=a-b .
$$

We define a Fourier transform on $L^{\prime}$ by

$$
\hat{\Psi}[u, v]=\iint \Psi\left[u^{\prime}, v^{\prime}\right] \psi_{0}\left(\frac{u^{\prime} v-v^{\prime} u}{2}\right) d u^{\prime} d v^{\prime} .
$$


The space $V_{L^{\prime}}$ corresponding to $V$ is actually identical to $V$. We can take

$$
P(X)=\int \Psi[u, v]|u v|^{s} d u d v / L\left(s, 1_{F}\right)^{2}
$$

where $\Psi$ is in $V_{L}$. The Tate functional equation reads

$$
\int \Psi[u, v]|u v|^{s} d u d v / L\left(s, 1_{F}\right)^{2}=\int \hat{\Psi}[u, v]|u v|^{1-s} d u d v / L\left(1-s, 1_{F}\right)^{2}
$$

This shows that $P$ is indeed symmetric for $\Psi \in V_{L^{\prime}}$. Now $V_{L^{\prime}}$ is spanned by functions of the form

$$
\Psi(x, y)=\phi(x) \hat{\phi}(y),
$$

where $\phi$ is a Schwartz-Bruhat function such that

$$
\phi(u x)=\phi(x)
$$

for $|u|=1$. For such a $\Psi$, the polynomial $P$ has the form

$$
P(X)=S(X) S\left(X^{-1} q^{-1}\right)
$$

where $S$ is an arbitrary Laurent polynomial. Any symmetric polynomial is a linear combination of polynomials of this form, and our assertion follows in this case.

4.8. We pass to the study of the polynomials $Q$ in Lemma 4.9. Here $T$ is the multiplicative group of $L$, the algebra of matrices of the form

$$
\left(\begin{array}{cc}
a & b \tau \tau_{0} \\
b & a
\end{array}\right)
$$

The character $\eta \circ \operatorname{det}$ is a multiplicative character $\eta_{L}$ of $L$ and

$$
L(s, \eta) L\left(s, \eta_{\tau}\right)=L\left(s, \eta_{L}\right) .
$$

If $\tau=1$, then $L$ is the extension $E, \eta_{L}=1$, and we can apply the previous results. If $\tau=\tau_{0} \varepsilon^{2}$ where $\varepsilon$ and $\tau_{0}$ are units, then $\eta=\eta_{\tau}$ is an unramified character and the algebra $L$ is split; so we can again apply the previous results (with $X$ replaced by $-X)$.

If $|\tau|=1,\left|\tau_{0}\right|=q^{-1}$ or $|\tau|=q^{-1},\left|\tau_{0}\right|=1$, then $L$ is a ramified quadratic extension of $F$ but $\eta \circ$ det is an unramified character of $L$, so that we can apply again the previous results with $X$ replaced by $-X$. 
In the two remaining cases we have $|\tau|=\left|\tau_{0}\right|=q^{-1}$. Suppose first that $\tau=\tau_{0} \varepsilon^{2}$ where $\varepsilon$ is a unit. We may as well assume $\varepsilon=1$. Then $L$ is isomorphic to the sum of two copies of $F$. A function $\Phi(b, a)$ is identified to the function $\Psi(u, v)$ where

$$
u=a+b \tau_{0}, \quad v=a-b \tau_{0}
$$

The symplectic Fourier transform becomes the following Fourier transform on $L$ :

$$
\hat{\Psi}(u, v)=\int \Psi\left(u^{\prime}, v^{\prime}\right) \psi_{0}\left(\frac{u^{\prime} v-v^{\prime} u}{2 \tau_{0}}\right) d u^{\prime} d v^{\prime}
$$

The space $V_{L}$ corresponding to $V$ is the space of functions $\Psi$ such that

$$
\hat{\Psi}=\Psi, \quad \Psi(u x, u y)=\Psi(x, y) \quad \text { for } u \in R_{F}^{\times} .
$$

Since $\eta$ is ramified, the polynomial $P$ has the form

$$
P(X)=\int \Psi(u, v)|u v|^{s} \eta(u v) d u d v
$$

for $\Psi \in V_{L}$. Now the Tate functional equation reads

$$
\int \hat{\Psi}(u, v)|u v|^{1-s} \eta(u v) d^{\times} u d^{\times} v=\eta(-1) \varepsilon(\eta, s, \psi)^{2} \int \Psi(u, v)|u v|^{s} \eta(u v) d^{\times} u d^{\times} v
$$

where $\psi$ is the character of $F$ defined by

$$
\psi(x)=\psi_{0}\left(\frac{x}{2 \tau_{0}}\right)
$$

If $\phi$ is the function of support $R^{\times}$defined by

$$
\phi(u)=\eta(u)
$$

for $|u|=1$, its Fourier transform with respect to $\psi$ has the form $c \phi$ for a suitable $c$. The function

$$
\Psi(x, y)=c \phi(x) \phi(y)
$$

is thus in $V_{L}$, and the Tate functional equation in fact reads

$$
\int \hat{\Psi}(u, v)|u v|^{1-s} \eta(u v) d^{\times} u d^{\times} v=\int \Psi(u, v)|u v|^{s} \eta(u v) d^{\times} u d^{\times} v .
$$


For $\Psi \in V_{L}$ this is a symmetric polynomial in $X$. Moreover, just as before, this polynomial is arbitrary.

Finally, we assume $\tau=\varepsilon \tau_{0}$ where $\varepsilon$ is a unit which is not a square. Thus $L$ is another ramified extension of $F$. A function $\Phi$ on $F^{2}$ is identified to the function $\Psi$ on $L$ defined by

$$
\Psi\left(a+b \tau_{0} \sqrt{\varepsilon}\right)=\Phi(b, a)
$$

If we define

$$
\psi(z)=\psi_{0}\left(\operatorname{Tr}\left(\frac{z}{2 \tau_{0} \varepsilon}\right)\right)
$$

and

$$
\hat{\Psi}(z)=\int \Psi(u) \psi(u z) d u
$$

then the image $V_{L}$ of $V$ is the space of functions $\Psi$ such that

$$
\hat{\Psi}(z)=\Psi(\bar{z}), \quad \Psi(u z)=\Psi(z)
$$

for $u \in R_{F}^{\times}$. The character $\eta_{L}$ is defined by $\eta_{L}(z)=\eta(z \bar{z})$. The Tate functional equation reads

$$
\int \hat{\Psi}(z) \eta_{L}(z)|z|^{1-s} d^{\times} z=\varepsilon\left(s, \eta_{L}, \psi\right) \int \Psi(z) \eta_{L}(z)|z|^{s} d^{\times} z .
$$

We first show that the $\varepsilon$ factor is actually one. It suffices to find $\Psi \in V_{L}$ such that the integral

$$
\int \Psi(z) \eta_{L}(z)|z| d^{\times} z
$$

is a positive constant. We first choose the corresponding function $\Phi$. Let $\phi^{0}$ be the characteristic function of $R_{F}^{\times}$and $\phi_{0}$ the characteristic function of $R$. The Fourier transform of $\phi^{0}$ with respect to $\psi_{0}$ is the function $\phi_{1}$ defined by

$$
\phi_{1}(x)=\phi_{0}(x)-q^{-1} \phi_{0}(x \varpi)
$$

It follows that the function

$$
\Phi(x, y)=\phi_{1}(x) \phi^{0}(y)
$$


is in $V$. We consider the corresponding function $\Psi$ in $V_{L}$. Its support is contained in the set of units of $L$. Up to a positive factor, the corresponding Tate integral is found to be equal to

$$
1-q^{-1}-\sum_{x \in k^{x}} \eta\left(1-x^{2} \varepsilon\right)
$$

As we have seen in the previous section, the sum is actually 0 or -2 and so the total integral is $>0$, and our assertion is proved. We finish the proof as before: the polynomial $Q$ is the sum of two polynomials

$$
P(X)=\int \Psi(z) \eta_{L}(z)|z|^{s} d^{\times} z
$$

with $\Psi \in V_{L}$, and

$$
P_{1}(X)=X \int \Psi(z) \eta^{\prime}(z)|z|^{s} d^{\times} z
$$

with $\Psi \in V_{1 L}$, the space corresponding to $V_{1}$. Then $P$ (resp. $P_{1}$ ) is an arbitrary symmetric polynomial containing monomials of even (resp. odd) degree. Our assertion follows. This conclude the proof of the lemma and the proposition.

4.9. We conclude this section by the following corollary to Proposition 4.1:

LEMMA 4.12. If $\Phi$ and $\Phi^{\prime}$ have matching orbital integrals, then

$$
\left|\tau_{0}\right|^{s-1 / 2} \int \phi(x)|x|^{s} \eta(x) d^{\times} L(s, 1)=\int \phi^{\prime}(x)|x|^{s} d^{\times} x L(s, \eta) .
$$

This follows from the proof of the proposition.

\section{Global theory.}

5.1. We let $E / F$ be a quadratic extension of number fields. Then $S$ is an algebraic variety defined over $F$. We set

$$
g^{*}=w^{t} \bar{g} w
$$

Then $G=G L(2, E)$ operates on $S$ on the right:

$$
s \mapsto g^{*} s g .
$$

For $\varepsilon$ in $S$ we denote by $H_{\varepsilon}^{0}, H_{\varepsilon}$, and $\lambda_{\varepsilon}$ the corresponding unitary group, similitude group, and similitude ratio. Thus for $h \in H_{\varepsilon}$,

$$
h^{*} \varepsilon h=\lambda_{\varepsilon}(h) \varepsilon
$$


We say that two matrices $s_{1}$ and $s_{2}$ are rationally equivalent over $F$ if there is $\gamma \in G L(2, E)$ such that

$$
s_{1}=\gamma^{*} s_{2} \gamma
$$

We say they are projectively equivalent if there is a $\gamma \in G L(2, E)$ and a $\mu \in F^{\times}$such that

$$
s_{1}=\mu \gamma^{*} s_{2} \gamma
$$

In particular, $\mu$ is in the image $F^{\varepsilon}$ of $\lambda_{\varepsilon}$ if and only if $\mu \varepsilon$ and $\varepsilon$ are rationally equivalent. Two matrices are rationally (resp. projectively) equivalent if and only if they are rationally (resp. projectively) equivalent at each place of $F$ which is inert in $E$.

At a nonarchimedean place, the two definitions of equivalence coincide. Moreover, $s_{1}$ is equivalent to $s_{2}$ if and only if det $s_{1}$ and det $s_{2}$ are equal up to the product by a norm. In particular, $s$ belongs to the orbit of $e$, that is, the form associated to $s w$ is split, if and only if det $s$ is a norm.

At a real place $v$ of $F$ inert in $E, s_{1}$ and $s_{2}$ are projectively equivalent if and only if det $s_{1}$ and det $s_{2}$ are equal up to the product by a norm, that is, have the same sign. Matrices $s$ with positive determinant are those for which $s w$ is split; they form a single rational equivalence class. The set of matrices with negative determinant is the union of two rational classes.

For a place $v$ of $F$ inert in $E$, set $F_{v}^{\varepsilon}=\lambda_{\varepsilon}\left(H_{\varepsilon}\left(F_{v}\right)\right)$. Then $F_{v}^{\varepsilon}$ is actually equal to $F_{v}^{\times}$, except when $v$ is real and inert, and det $\varepsilon_{v}<0$, in which case it consists of the positive elements of $F_{v}{ }^{\times}$.

Let $F_{\mathbb{A}}^{\varepsilon}$ be the product of the $F_{v}^{\varepsilon}$. Thus

$$
F_{\mathbb{A}}^{\varepsilon}=\lambda_{\varepsilon}\left(H_{\varepsilon}\left(F_{\mathbb{A}}\right)\right)
$$

Clearly, $F_{\AA}^{\varepsilon}$ is an open subgroup of finite index in $F_{\mathbb{A}}^{\times}$. Since $F^{\times}$is dense in the product of the $F_{v}^{\times}$with $v$ real, we have

$$
F_{\mathbb{A}}^{\times}=F^{\times} F_{\mathbb{A}}^{\varepsilon} .
$$

On the other hand,

$$
F^{\varepsilon}=F^{\times} \cap F_{\AA}^{\varepsilon}
$$

It follows that $F^{\varepsilon}$ is a subgroup of finite index in $F^{\times}$, and we have a disjoint union

$$
F_{\mathbb{A}}^{\times}=\bigcup_{\xi \in F^{\times} / F^{\varepsilon}} \xi F_{\AA}^{\varepsilon} .
$$


We have exact sequences

$$
\begin{aligned}
& 1 \rightarrow H_{\varepsilon}^{0}(F) \rightarrow H_{\varepsilon}(F) \stackrel{\lambda_{\varepsilon}}{\rightarrow} F^{\varepsilon} \rightarrow 1, \\
& 1 \rightarrow H_{\varepsilon}^{0}\left(F_{v}\right) \rightarrow H_{\varepsilon}\left(F_{v}\right) \stackrel{\lambda_{\varepsilon}}{\rightarrow} F_{v}^{\varepsilon} \rightarrow 1, \\
& 1 \rightarrow H_{\varepsilon}^{0}\left(F_{\mathbb{A}}\right) \rightarrow H_{\varepsilon}\left(F_{\mathbb{A}}\right) \stackrel{\lambda_{\varepsilon}}{\rightarrow} F_{\mathbb{A}}^{\varepsilon} \rightarrow 1 .
\end{aligned}
$$

We choose a measure on $H_{\varepsilon}\left(F_{\mathrm{A}}\right)$ that we write as a product of local measures. It is assumed that for almost all places the measure of the maximal compact defined below is 1 . We choose a measure on $F_{v}^{\times}$in the usual way. It induces a measure on $F_{v}^{\varepsilon}$, and we use the local sequence to define a measure on $H_{\varepsilon}^{0}\left(F_{v}\right)$.

We choose a system of representatives $\{\varepsilon\}$ for the rational classes. We can take it to be of the form $\varepsilon=\mu \varepsilon^{\prime}$ where $\left\{\varepsilon^{\prime}\right\}$ is a set of representatives for the projective classes and, for each $\varepsilon^{\prime},\{\mu\}$ is a set of representatives for the cosets of $F^{\varepsilon^{\prime}}$ in $F^{\times}$. We choose a function of compact support $\Phi$ on $S\left(F_{\mathbb{A}}\right)$. We assume that $\Phi$ is a product of local functions $\Phi_{v}$. Thus $\Phi_{v}$ is a smooth function of compact support on $S\left(F_{v}\right)$. Furthermore, for almost all $v$, it is in fact the characteristic function of the set of integral elements of $S_{v}$ with unit determinant. For each $\varepsilon$, we choose a function $f_{\varepsilon}$ on $G L\left(2, E_{\mathbb{A}}\right)$, smooth of compact support, such that

$$
\int_{H_{0}^{\varepsilon}\left(F_{\mathrm{A}}\right)} f_{\varepsilon}\left(h_{\varepsilon} g\right) d h_{\varepsilon}=\Phi\left(g^{*} \varepsilon g\right) .
$$

In order for this integral to be nonzero, there must be an idèle $z$ which is a norm at each place such that $z$ det $\varepsilon$ is in the image of the support of $\Phi$ under det. Let $X(\Phi)$ be the set of $\varepsilon$ with this property. This image is a compact set; since $N_{E / F}\left(E_{\mathbb{A}}^{\times}\right)$ is open in $F_{\mathbb{A}}^{\times}$, this image is contained in a finite union of cosets of $N_{E / F}\left(E_{\mathbb{A}}^{\times}\right)$. A fortiori, det $\varepsilon$ belongs to a finite union of cosets of $N_{E / F}\left(E^{\times}\right)$. Writing $\varepsilon=\mu \varepsilon^{\prime}$, we see that $\varepsilon^{\prime}$ belongs to a finite set. Thus $X(\Phi)$ is a finite set itself.

It will be convenient to choose a finite set $X$ of $\varepsilon$ and to choose the function $\Phi_{v}$ for each place $v$ of $F$ inert in $E$ with support in the union of the orbits of the $\varepsilon \in X$ under $G L\left(2, E_{v}\right)$. Then

$$
\Phi\left(g^{*} \varepsilon g\right)=0
$$

if $\varepsilon$ is not in $X$. For $\varepsilon \notin X$ we will take $f_{\varepsilon}=0$.

Next, to each $\varepsilon$ we associate a kernel function

$$
K_{\varepsilon}\left(g_{1}, g_{2}\right)=\sum_{\xi \in G L(2, E)} f_{\varepsilon}\left(g_{1}^{-1} \xi g_{2}\right) .
$$

We will set

$$
K_{\varepsilon}(g)=\int_{H_{\varepsilon}(F) \backslash H_{\varepsilon}\left(F_{\mathrm{A}}\right)} K_{\varepsilon}(h, g) d h .
$$


We claim that

$$
\sum_{\varepsilon} K_{\varepsilon}(g)=\int_{F_{A}^{\times} / F^{\times}} d^{\times} z \sum_{\xi \in S(F)} \Phi\left(g^{*} \xi z g\right) .
$$

Indeed, it suffices to prove this for $g=e$. We can write

$$
K_{\varepsilon}(e)=\sum_{\xi \in H_{\varepsilon}(F) \backslash G(F)} \int\left(\int f_{\varepsilon}\left(h_{0} h_{1} \xi\right) d h_{0}\right) d h_{1}
$$

with

$$
h_{0} \in H_{\varepsilon}^{0}\left(F_{\mathrm{A}}\right), \quad h_{1} \in H_{\varepsilon}^{0}\left(F_{\mathrm{A}}\right) \backslash H_{\varepsilon}\left(F_{\mathrm{A}}\right) .
$$

Thus we find

$$
\begin{aligned}
K_{\varepsilon}(e) & =\sum_{\xi} \int \Phi\left(\xi^{*} \varepsilon \xi \lambda\left(h_{1}\right)\right) d h_{1} \\
& =\sum_{\xi} \int_{F_{\AA}^{\varepsilon}} \Phi\left(\xi^{*} \varepsilon \xi z\right) d^{\times} z .
\end{aligned}
$$

Replacing $\varepsilon$ by $\mu \varepsilon$ does not change the similitude group; thus summing over $\mu \in F^{\times} / F^{\varepsilon}$, we find

$$
\begin{aligned}
\sum_{\mu} K_{\mu \varepsilon}(e) & =\sum_{\xi} \sum_{\mu} \int_{F_{A}^{\varepsilon}} \Phi\left(\xi^{*} \varepsilon \mu z \xi\right) d^{\times} z \\
& =\sum_{\xi} \int_{F_{A}^{\times}} \Phi\left(\xi^{*} \varepsilon z \xi\right) d^{\times} z \\
& =\int_{F_{A}^{\times} / F^{\times}} \sum_{\xi} \sum_{\mu \in F^{\times}} \Phi\left(\xi^{*} \mu \varepsilon z \xi\right) d^{\times} z \\
& =\int_{F_{A}^{\times} / F^{\times}} \sum_{\sigma} \Phi(\sigma z) d^{\times} z
\end{aligned}
$$

where the sum is for $\sigma$ in the projective class of $\varepsilon$. Summing over the projective classes, we find our result.

5.2. We set

$$
K_{\Phi}(g)=\sum_{\varepsilon} K_{\varepsilon}(g)
$$


We have thus

$$
K_{\Phi}(g)=\int_{F_{\mathrm{A}}^{\times} / F^{\times}} \sum_{\xi \in S(F)} \Phi\left(g^{*} \xi z g\right) d^{\times} z .
$$

Associated to the function $f_{\varepsilon}$ and the trivial central character, we have in the usual way a cuspidal kernel, a special kernel, and an Eisenstein kernel, $K_{\varepsilon}^{\text {cusp }}, K_{\varepsilon}^{\text {spe }}$, and $K_{\varepsilon}^{e i s}$, respectively. We define

$$
K_{\Phi}^{c u s p}(g)=\sum_{\varepsilon} \int K_{\varepsilon}^{c u s p}\left(h_{\varepsilon}, g\right) d h_{\varepsilon}
$$

the integral over $Z\left(F_{\mathbb{A}}\right) H_{\varepsilon}(F) \backslash H_{\varepsilon}\left(F_{\mathbb{A}}\right)$. We define similarly $K_{\Phi}^{\text {spe }}, K_{\Phi}^{\text {eis }}$. We remark that for any cusp form $\phi$ on $G L\left(2, E_{\mathbb{A}}\right)$, invariant under the center,

$$
\int K_{\Phi}(g) \phi(g) d g=\int K_{\Phi}^{\text {cusp }}(g) \phi(g) d g
$$

Thus we may view $K_{\Phi}^{\text {cusp }}$ as the projection of $K_{\Phi}$ on the space of cusp forms.

In this section, our goal is to compute the integral

$$
I_{\Phi}^{\text {cusp }}(s)=\int K_{\Phi}^{\text {cusp }}(h) E(h, s) \eta(\lambda(h)) d h
$$

where $E(g, s)$ is an Eisenstein series on the group $H=H_{w}$, suitably normalized. The integral is over $Z\left(F_{\mathbb{A}}\right) H(F) \backslash H\left(F_{\mathbb{A}}\right)$. We first compute formally. We will justify our steps completely under the restrictions mentioned in the introduction.

At this point, we assume that every real place of $F$ splits in $E$ and that every finite place of even residual characteristic splits in $E$. We need to specify the measures we are using on $H$ and $G^{\prime}$, the maximal compacts, and the functions $f, f^{\prime}$ which are used to define the Eisenstein series. For each place $v$ of $F$ inert in $E$, we choose an element $t_{v}$ such that

$$
E_{v}=F_{v}\left(\sqrt{t_{v}}\right)
$$

If $v$ is unramified in $E$, we take for $t_{v}$ a unit; if $v$ is ramified, we take for $t_{v}$ a uniformizer of $F_{v}$. For $v$ split, we take $t_{v}=1$. We set

$$
t_{0}=\prod t_{v} .
$$

Then for $v$ inert, conjugacy by

$$
g_{v}=\left(\begin{array}{cc}
\sqrt{t_{v}} & 0 \\
0 & 1
\end{array}\right)
$$


defines an isomorphism

$$
\xi_{v}: H_{v} \rightarrow G_{v}^{\prime} Z_{v}
$$

If $v$ is split, then $H_{v}$ is the set of triples $\left(g_{1}, g_{2}, \lambda\right)$ with $g_{i} \in G_{v}^{\prime}, \lambda \in F^{\times}$such that

$$
g_{1}=w^{t} g_{2}^{-1} w \lambda
$$

On the other hand, $G_{v}^{\prime} Z_{v}$ is isomorphic to the set of pairs

$$
\left(g \lambda_{1}, g \lambda_{2}\right)
$$

with $g \in G_{v}, \lambda_{i} \in F_{v} \times$. Thus we have again an isomorphism

$$
\xi_{v}: H_{v} \rightarrow G_{v}^{\prime} Z_{v}
$$

The product of the $\xi_{v}$ defines then an isomorphism

$$
\xi: H\left(F_{\mathbb{A}}\right) \rightarrow G^{\prime}\left(F_{\mathbb{A}}\right) Z\left(F_{\mathbb{A}}\right) .
$$

Recall $P$ is the group of upper triangular matrices, $P_{H}$ its intersection with $H$, and $P^{\prime}$ its intersection with $G^{\prime}$. Here it will be more convenient to think of $P$ as a group defined over $E$. For each finite place $v$ of $F$ we set

$$
K_{v}^{\prime}=G L\left(2, R_{v}\right)
$$

For $v$ real we set

$$
K_{v}^{\prime}=O(2, \mathbb{R})
$$

For $v$ complex we set

$$
K_{v}^{\prime}=U(2, \mathbb{C})
$$

We regard $Z \simeq E^{\times}$as an algebraic group over $F$, and for each place $v$ of $F$ we denote by $U_{v}$ the maximal compact subgroup of $Z_{v}$. We let $K^{\prime}$ be the product of the $K_{v}^{\prime}$ and $U$ the product of the $U_{v}$. We define a function $f^{\prime}$ on $G^{\prime}\left(F_{\mathbb{A}}\right) Z\left(F_{\mathbb{A}}\right)$ by

$$
f^{\prime}\left(g^{\prime} z\right)=|t|^{s}
$$

for

$$
g^{\prime}=\left(\begin{array}{ll}
t & u \\
0 & 1
\end{array}\right) k^{\prime}
$$


Thus we may write $f^{\prime}$ as the product of local functions $f_{v}^{\prime}$ which, for $v$ inert, are the functions introduced in the previous sections. We use the isomorphism $\xi$ to transport $K^{\prime} U$ and $f^{\prime}$ to $H$. We denote by $K_{H}$ and $f$ the corresponding maximal subgroup and function. Thus

$$
f(h, s)=|t|^{s} \quad \text { if } h=\left(\begin{array}{cc}
t z & x \\
0 & z
\end{array}\right) k
$$

Then

$$
E(h, s)=\sum_{\gamma \in P_{H}(F) \backslash H(F)} f(\gamma h, s) .
$$

On $G^{\prime}\left(F_{\mathbb{A}}\right) / Z^{\prime}\left(F_{\mathbb{A}}\right)$, we choose a measure compatible with the Iwasawa decomposition

$$
d g^{\prime}=d k^{\prime}|t|^{-1} d^{\times} t d u
$$

if

$$
g^{\prime}=\left(\begin{array}{cc}
t & u \\
0 & 1
\end{array}\right) k^{\prime}
$$

Here $d^{\times} t$ and $d u$ are the Tamagawa measures. We transport this measure to $H\left(F_{\mathbb{A}}\right) / Z\left(F_{\mathbb{A}}\right)$. The measure needs not be the same as the one we have used to define the kernels in terms of $\Phi$. The integral (33) is defined for $\mathfrak{R} s>1$. For simplicity, we will drop the index $\Phi$ from the kernels. Unfolding the Eisenstein series, we find also for $\mathfrak{R} s>1$

$$
I^{\text {cusp }}(s)=\int_{Z\left(F_{\AA}\right) P_{H}(F) \backslash H\left(F_{\AA}\right)} K^{c u s p}(h) f(h, s) \eta(\lambda(h)) d h .
$$

We have

$$
K^{c u s p}=K-K^{e i s}-K^{s p e}, \quad K=K^{r e g}+K^{\text {sing }}
$$

where

$$
\begin{aligned}
& K^{r e g}(g)=\int \sum_{\xi \notin P(E) \cap S(F)} \Phi\left(g^{*} \xi z g\right) d^{\times} z, \\
& K^{\text {sing }}(g)=\int \sum_{\xi \in P(E) \cap S(F)} \Phi\left(g^{*} \xi z g\right) d^{\times} z .
\end{aligned}
$$


Each function of $g$ is invariant under $P(E)$ on the left. It will be convenient to introduce the function $\tilde{K}$ defined by

$$
\tilde{K}=K^{s i n g}-K^{e i s}-K^{s p e} .
$$

Then

$$
K^{\text {cusp }}=K^{r e g}+\tilde{K}
$$

We will denote by $\tilde{I}$ the integral

$$
\tilde{I}(s)=\int_{Z\left(F_{\AA}\right) P_{H}(F) \backslash H\left(F_{\AA}\right)} \tilde{K}(h) f(h, s) \eta(\lambda(h)) d h .
$$

We will define similarly $I^{e i s}, I^{\text {reg }}$, and so on. Thus

$$
I^{c u s p}(s)=I^{r e g}(s)+\tilde{I}(s) .
$$

5.3. We first consider $I^{\text {reg }}$. We need the following lemma which relates conjugation under $P_{H}(F)$ and $H(F)$ in $S(F)$ :

Lemma 5.1. Suppose $\xi \notin P(E) \cap S(F)$. Then $\xi$ is conjugate to an element of $S_{0}(F)$ by an element of $P_{H}(F)$. Moreover, two elements of $S_{0}(F)$ which are not in $P(F)$ are conjugate under $H(F)$ if and only if they are conjugate under $P_{H}(F)$. Finally, if $\xi$ is in $S_{0}(F)-S_{0}(F) \cap P(E)$, then its centralizer in $P_{H}(F)$ is equal to $Z(F)$.

According to the lemma, a semisimple element of $S(F)-S(F) \cap P(E)$ can be written in one way in the form

$$
\gamma^{-1} z\left(\begin{array}{cc}
1 & \beta \\
1 & 1
\end{array}\right) \gamma, \quad \gamma \in P_{H}(F) / Z(F), \quad z \in F^{\times}, \quad \beta \in F-\{0,1\},
$$

or in the form

$$
\gamma^{-1} z\left(\begin{array}{ll}
0 & \beta \\
1 & 0
\end{array}\right) \gamma, \quad \gamma \in P_{H}(F) / Z(F), \quad z \in F^{\times}, \quad \beta \in F^{\times} / F^{\times 2} .
$$

A unipotent element in $S(F)-S(F) \cap P(E)$ can be written in one way in the form

$$
\gamma^{-1} z\left(\begin{array}{ll}
1 & 0 \\
1 & 1
\end{array}\right) \gamma, \quad \gamma \in P_{H}(F) / Z(F), \quad z \in F^{\times} .
$$

Thus the integral $I^{r e g}(s)$ can be written as a sum

$$
I^{r e g}(s)=\sum_{\beta \in F-\{0,1\}} \Omega(\Phi, \beta, s)+\sum_{\beta \in F^{\times} / F^{\times 2}} \Omega^{\infty}(\Phi, \beta, s)+I^{u n i}(\Phi, s)
$$


where we have set

$$
\begin{gathered}
\Omega(\Phi, \beta, s)=\int \Phi\left[h^{-1} z\left(\begin{array}{ll}
1 & \beta \\
1 & 1
\end{array}\right) h\right] \eta(\lambda(h)) f(h, s) d h d^{\times} z, \\
\Omega^{\infty}(\Phi, \beta, s)=\int \Phi\left[h^{-1} z\left(\begin{array}{ll}
0 & \beta \\
1 & 0
\end{array}\right) h\right] \eta(\lambda(h)) f(h, s) d h d^{\times} z,
\end{gathered}
$$

and

$$
I^{u n i}(\Phi, s)=\int \Phi\left[h^{-1} z\left(\begin{array}{ll}
1 & 0 \\
1 & 1
\end{array}\right) h\right] \eta(\lambda(h)) f(h, s) d h d^{\times} z
$$

where the integral is for $h \in Z\left(F_{\mathbb{A}}\right) \backslash H\left(F_{\mathbb{A}}\right)$ and $z \in F_{\mathbb{A}}^{\times}$.

We give another formula for the last integral: we can change $h$ to $w h$ to get

$$
I^{u n i}(s)=\int \Phi\left[h^{-1} z\left(\begin{array}{ll}
1 & 1 \\
0 & 1
\end{array}\right) h\right] \eta(\lambda(h)) f(w h, s) d h d^{\times} z
$$

Integrating via the Iwasawa decomposition, we get

$$
I^{u n i}(s)=m(s) \int_{F_{\Lambda}^{\times} \times K \times F_{a}^{\times}} \Phi\left[k^{-1} z\left(\begin{array}{cc}
1 & t \\
0 & 1
\end{array}\right) k\right] \eta(t)|t|^{s} \eta(\lambda(k)) d^{\times} t d k d^{\times} z
$$

where we have set

$$
m(s)=\int_{\sqrt{\tau_{0}} F_{\mathrm{A}}} f\left[w\left(\begin{array}{cc}
1 & x \\
0 & 1
\end{array}\right) z k\right] d u
$$

here $E=F\left(\sqrt{\tau_{0}}\right)$ and

$$
x=\frac{u}{\sqrt{t_{0}}} .
$$

For future needs, we compare $m(s)$ with the scalar $m^{\prime}(s)$ defined by

$$
m^{\prime}(s)=\int_{F_{A}} f^{\prime}\left[w\left(\begin{array}{ll}
1 & u \\
0 & 1
\end{array}\right)\right] d u
$$

We find

$$
m(s)=\left|t_{0}\right|^{s} m^{\prime}(s)
$$


As in the local case, we introduce a Schwartz-Bruhat function $\phi$ on $F_{\mathbb{A}}$ :

$$
\phi(x)=\int \Phi\left[k^{-1} z\left(\begin{array}{ll}
1 & x \\
0 & 1
\end{array}\right) k\right] \eta(\lambda(k)) d^{\times} z d k
$$

Then

$$
I^{u n i}(s)=m(s) \int \phi(t) \eta(t)|t|^{s} d^{\times} t .
$$

5.4. We now consider the integral $\tilde{I}$. Recall

$$
\tilde{I}(s)=\int_{Z\left(F_{\mathrm{A}}\right) P_{H}(F) \backslash H\left(F_{\mathrm{A}}\right)} \tilde{K}(h) f(h, s) \eta(\lambda(h)) d h .
$$

The integral $\tilde{I}$ factors through an integral on $N_{H}(F) \backslash N_{H}\left(F_{\mathrm{A}}\right)$, namely,

$$
\int_{u \in F_{\mathrm{A} / F}} \tilde{K}\left[\left(\begin{array}{ll}
1 & x \\
0 & 1
\end{array}\right) g\right] d u
$$

Let us agree to denote by $d x$ the measure on $\sqrt{\tau_{0}} F_{\mathbb{A}}$ such that

$$
\int_{\sqrt{\tau_{0}} F_{\mathrm{A}} / \sqrt{\tau_{0}} F} d x=1 \text {. }
$$

Thus we can write $x=\sqrt{\tau_{0}} v$ and $d x=d v$ where $d v$ is the Tamagawa measure on $F_{\mathbb{A}}$. In terms of the variable $u$ defined by

$$
x=\frac{u}{\sqrt{t_{0}}}
$$

we have

$$
v=\frac{u}{\sqrt{\tau_{0} t_{0}}}
$$

so that

$$
d x=d v=\left|t_{0}^{-1 / 2}\right| d u
$$

Let $\psi_{0}$ be a nontrivial additive character of $E_{\mathbb{A}}$ trivial on $E+F_{\mathbb{A}}$. If $\mu$ is a smooth function on $E_{\mathbb{A}} / E$, then

$$
\int_{\sqrt{\tau_{0}} F_{\mathrm{A}} / \sqrt{\tau_{0} F}} \mu(x) d x=\int_{E_{\mathrm{A}} / E} \mu(y) d y+\sum_{\alpha \in F^{\times}} \int_{E_{\mathrm{A} / E}} \mu(y) \psi_{0}(\alpha y) d y,
$$


A TRACE FORMULA FOR SYMMETRIC SPACES

355

where $d y$ is the Tamagawa measure on $E$, that is, the one for which the measure of $E_{\mathbb{A}} / E$ is 1 . Recall

$$
\tilde{K}(g)=K^{s i n g}(g)-K^{s p e c}(g)-K^{e i s}(g) .
$$

The sum

$$
-\sum_{\alpha \in F^{\times}} \int_{E_{\mathbf{A}} / E} K^{s p e}\left[\left(\begin{array}{ll}
1 & y \\
0 & 1
\end{array}\right) g\right] \psi_{0}(\alpha y) d y
$$

vanishes. Thus we can evaluate (41) as follows:

$$
\begin{gathered}
\left|t_{0}\right|^{1 / 2} \int_{\sqrt{\tau_{0} F_{\mathrm{A}} / \sqrt{\tau_{0}} F}} K^{s i n g}\left[\left(\begin{array}{ll}
1 & x \\
0 & 1
\end{array}\right) g\right] d x, \\
-\left|t_{0}\right|^{1 / 2} \int_{E_{\mathrm{A} / E}}\left(K^{s p e c}+K^{e i s}\right)\left[\left(\begin{array}{ll}
1 & y \\
0 & 1
\end{array}\right) g\right] d y, \\
-\left|t_{0}\right|^{1 / 2} \sum_{\alpha \in F^{\times}} \int_{E_{\mathrm{A} / E}} K^{e i s}\left[\left(\begin{array}{ll}
1 & y \\
0 & 1
\end{array}\right) g\right] \psi_{0}(\alpha y) d y .
\end{gathered}
$$

Because of the cuspidality, we can replace $K^{\text {sp }}+K^{\text {ers }}$ by $K$ in the middle integral. Thus the integral (41) can be written as the sum of three terms:

$$
\begin{gathered}
\left|t_{0}\right|^{1 / 2} \int_{\sqrt{\tau_{0} F_{\mathrm{A}} / \sqrt{\tau_{0} F}}} K^{\operatorname{sing}}\left[\left(\begin{array}{ll}
1 & x \\
0 & 1
\end{array}\right) g\right] d x, \\
-\left|t_{0}\right|^{1 / 2} \int_{E_{\mathrm{A} / E}} K\left[\left(\begin{array}{ll}
1 & y \\
0 & 1
\end{array}\right) g\right] d y, \\
-\left|t_{0}\right|^{1 / 2} \sum_{\alpha \in F^{\times}} \int_{E_{\mathrm{A} / F}} K^{e i s}\left[\left(\begin{array}{ll}
1 & y \\
0 & 1
\end{array}\right) g\right] \psi_{0}(\alpha y) d y .
\end{gathered}
$$

To evaluate (45) we write

$$
K^{\text {sing }}(g)=\sum_{\beta \in F} \int \Phi\left[g^{*}\left(\begin{array}{cc}
1 & \beta \\
0 & 1
\end{array}\right) z g\right] d^{\times} z+\sum_{\beta \in F,\{\alpha \neq \bar{\alpha}\} / F^{\times}} \int \Phi\left[g^{*}\left(\begin{array}{cc}
\alpha & \beta \\
0 & \bar{\alpha}
\end{array}\right) z g\right] d^{\times} z .
$$

Integrating over $N_{H}(F) \backslash N_{H}\left(F_{\AA}\right)$, we get for (45)

$$
\begin{aligned}
& \left|t_{0}\right|^{1 / 2} \sum_{\beta \in F} \int \Phi\left[g^{*}\left(\begin{array}{ll}
1 & \beta \\
0 & 1
\end{array}\right) z g\right] d^{\times} z \\
+ & \left|t_{0}\right|^{1 / 2} \sum_{\beta \in F,\{\alpha \neq \bar{\alpha}\} / F^{\times}} \int \Phi\left[g^{*}\left(\begin{array}{cc}
\alpha & \beta+(\alpha-\bar{\alpha}) \sqrt{\tau_{0}} v \\
0 & \bar{\alpha}
\end{array}\right) z g\right] d^{\times} z d v
\end{aligned}
$$


where $d v$ is the Tamagawa measure on $F_{\mathbb{A}} / F$. The second term simplifies, and we obtain for (45) the expression

$$
\begin{aligned}
& \left|t_{0}\right|^{1 / 2} \sum_{\beta \in F} \int_{F_{\AA}^{\times}} \Phi\left[g^{*}\left(\begin{array}{ll}
1 & \beta \\
0 & 1
\end{array}\right) z g\right] d^{\times} z \\
& +\left|t_{0}\right|^{1 / 2} \sum_{\{\alpha \neq \bar{\alpha}\} / F^{\times}} \int_{F_{\Lambda}^{\times} \times F_{\Lambda}} \Phi\left[g^{*}\left(\begin{array}{ll}
\alpha & v \\
0 & \bar{\alpha}
\end{array}\right) z g\right] d^{\times} z d v .
\end{aligned}
$$

Now we write again $K=K^{\text {sing }}+K^{\text {reg }}$ to compute (46). The integral of $-K^{\text {sing }}$ gives

$$
\begin{aligned}
& -\left|t_{0}\right|^{1 / 2} \sum_{\beta} \int \Phi\left[g^{*}\left(\begin{array}{cc}
1 & \beta+y+\bar{y} \\
0 & 1
\end{array}\right) z g\right] d^{\times} z d y \\
& -\left|t_{0}\right|^{1 / 2} \sum_{\beta \in F,\{\alpha \neq \bar{\alpha}\} / F^{\times}} \int \Phi\left[g^{*}\left(\begin{array}{cc}
\alpha & \beta+\alpha y+\overline{\alpha y} \\
0 & \bar{\alpha}
\end{array}\right) z g\right] d^{\times} z d y .
\end{aligned}
$$

This simplifies to

$$
\begin{aligned}
& -\left|t_{0}\right|^{1 / 2} \int_{F_{\mathrm{A}}} \Phi\left[g^{*}\left(\begin{array}{cc}
1 & x \\
0 & 1
\end{array}\right) z g\right] d^{\times} z d x \\
& -\left|t_{0}\right|^{1 / 2} \sum_{\{\alpha \neq \bar{\alpha}\} / F^{\times}} \int_{F_{\mathrm{A}}} \Phi\left[g^{*}\left(\begin{array}{ll}
\alpha & x \\
0 & \bar{\alpha}
\end{array}\right) z g\right] d^{\times} z d x
\end{aligned}
$$

where $d x$ is the Tamagawa measure on $F_{\mathbb{A}}$. If we combine it with the expression we obtained for (45), we obtain

$$
\left|t_{0}\right|^{1 / 2} \sum_{\beta \in F} \int_{F_{\mathrm{A}}} \Phi\left[g^{*}\left(\begin{array}{ll}
1 & \beta \\
0 & 1
\end{array}\right) z g\right] d^{\times} z-\left|t_{0}\right|^{1 / 2} \int_{F_{\mathrm{A}}} \Phi\left[g^{*}\left(\begin{array}{ll}
1 & x \\
0 & 1
\end{array}\right) z g\right] d^{\times} z d x
$$

It remains to compute the integral of $-K^{r e g}$ on $N\left(E_{\mathbb{A}}\right) / N(E)$. The matrices of the form

$$
\left(\begin{array}{ll}
0 & \beta \\
\gamma & 0
\end{array}\right)
$$

with $\beta, \gamma \in F^{\times}$form a set of representatives for the orbits of $N(E)$ on $S(F)-S(F) \cap$ $P(E)$. It follows that the integral of $-K^{r e g}$ is

$$
-\left|t_{0}\right|^{1 / 2} \sum_{\gamma \in F^{\times}} \int_{N\left(E_{A}\right)} \Phi\left[g^{*} n^{*} z\left(\begin{array}{ll}
0 & 1 \\
\gamma & 0
\end{array}\right) n g\right] d n d^{\times} z
$$


To compute (47) we set

$$
K^{w h i}(g)=\int_{E_{\mathrm{A} / E}} K^{e i s}\left[\left(\begin{array}{ll}
1 & x \\
0 & 1
\end{array}\right) g\right] \psi_{0}(x) d x d^{\times} z,
$$

and we see that (41) is equal to the sum of three terms

$$
\begin{aligned}
& \left|t_{0}\right|^{1 / 2}\left\{\sum_{\beta \in F} \int \Phi\left[g^{*}\left(\begin{array}{ll}
1 & \beta \\
0 & 1
\end{array}\right) z g\right] d^{\times} z\right. \\
& \left.-\int_{F_{A}} \Phi\left[g^{*}\left(\begin{array}{ll}
1 & y \\
0 & 1
\end{array}\right) z g\right] d^{\times} z d y\right\} \\
& -\left|t_{0}\right|^{1 / 2} \sum_{\gamma \in F^{\times}} \int_{N\left(E_{A}\right)} \Phi\left[g^{*} n^{*} z\left(\begin{array}{ll}
0 & 1 \\
\gamma & 0
\end{array}\right) n g\right] d n d^{\times} z \\
& -\left|t_{0}\right|^{1 / 2} \sum_{\alpha \in F^{\times}} K^{w h i}\left[\left(\begin{array}{ll}
\alpha & 0 \\
0 & 1
\end{array}\right) g\right] .
\end{aligned}
$$

For each integral, $z$ is integrated over $F_{\mathbb{A}}^{\times}$. To compute $\tilde{I}(s)$ we need to integrate each one of the above functions on $Z\left(F_{\mathbb{A}}\right)\left(N_{H}\right)\left(F_{\mathbb{A}}\right) P_{H}(F) \backslash H\left(F_{\mathbb{A}}\right)$ against $f(h, s) \eta(\lambda(h))$. To do so, we use the Iwasawa decomposition: we write

$$
h=\left(\begin{array}{ll}
t & 0 \\
0 & 1
\end{array}\right) k
$$

Then

$$
d h=|t|^{-1} d^{\times} t d k, \quad \eta(\lambda(h)) f(h, s)=\eta(t)|t|^{s} .
$$

We obtain $\tilde{I}$ as the sum of three new integrals $I_{i}, 1 \leqslant i \leqslant 3$. To compute $I_{1}(s)$ we recall the Schwartz-Bruhat function introduced earlier:

$$
\phi(x)=\int \Phi\left[k^{-1} z\left(\begin{array}{ll}
1 & z \\
0 & 1
\end{array}\right) k\right] \eta(\lambda(k)) d^{\times} z d k .
$$

We find

$$
I_{1}(s)=\left|t_{0}\right|^{1 / 2} \int_{F_{A}^{\times} / F^{\times}}\left[\sum_{\beta \in F} \phi\left(t^{-1} \beta\right)-\int \phi\left(t^{-1} x\right) d x\right] \eta(t)|t|^{s-1} d^{\times} t
$$

or, by the Poisson summation formula,

$$
\left|t_{0}\right|^{1 / 2} \int_{F_{\mathrm{A}}^{\times} / F^{\times}} \sum_{\beta \neq 0} \hat{\phi}(t \beta)|t|^{s} \eta(t) d^{\times} t
$$


Finally,

$$
I_{1}(s)=\left|t_{0}\right|^{1 / 2} \int_{F_{\wedge}^{\times}} \hat{\phi}(t)|t|^{s} \eta(t) d^{\times} t
$$

For $I_{2}(s)$ we find

$$
-\left|t_{0}\right|^{1 / 2} \int_{F_{\wedge}^{\times} / F^{\times}} \sum_{\gamma} \Phi\left[k^{-1} n^{*}\left(\begin{array}{cc}
0 & z \\
\gamma t^{2} z & 0
\end{array}\right) n k\right] \times \eta(\lambda(k)) \eta(t)|t|^{s+1} d^{\times} t d n d k d^{\times} z .
$$

This can be further transformed into (see $[\mathrm{JZ}]$ )

$$
I_{2}(s)=-\frac{1}{2}\left|t_{0}\right|^{1 / 2} \sum_{\omega^{2}=\eta} \int \Phi\left[k^{-1} n^{*}\left(\begin{array}{cc}
0 & z \\
z t & 0
\end{array}\right) n k\right] \omega(t)|t|^{s+1} \eta(\lambda(k)) d^{\times} t d n d k d^{\times} z
$$

The integral is for $z, t \in F_{\mathbb{A}}^{\times}, n \in N\left(E_{\mathbb{A}}\right), k \in K$, and the sum over all idèle-class characters $\omega$ of $F$ whose square is $\eta$. Finally, we have

$$
I_{3}(s)=-\left|t_{0}\right|^{1 / 2} \int \hat{F}_{\mathbb{A}}^{\times} K^{w h i}\left[\left(\begin{array}{ll}
t & 0 \\
0 & 1
\end{array}\right) k\right] \eta(t) \eta(\lambda(k))|t|^{s-1} d^{\times} t d k
$$

Thus we have

$$
I^{\text {cusp }}(s)=\sum_{\beta \in F-\{0,1\}} \Omega(\Phi, \beta, s)+\sum_{\beta \in F^{\times} / F^{\times 2}} \Omega^{\infty}(\Phi, \beta, s)+I^{u n i}(\Phi, s)+\sum_{i} I_{i}(s) .
$$

5.5. We consider similarly a smooth function of compact support $\Phi^{\prime}$ on $G^{\prime}\left(F_{\mathbb{A}}\right)$. We associate to $\Phi^{\prime}$ a kernel

$$
K^{\prime}(x, y)=\int_{F_{\mathfrak{A}}^{\times} / F^{\times}} \sum_{\xi \in G L(2, F)} \Phi^{\prime}\left(x^{-1} \xi z y\right) \eta(z) d^{\times} z
$$

and corresponding kernels $K^{\prime c u s p}, K^{\prime s p e}$, and $K^{\prime e i s}$. We consider the integral

$$
I^{\prime c u s p}(s)=\int K^{\prime c u s p}\left(g^{\prime}, g^{\prime}\right) E^{\prime}(g, s) d g^{\prime}
$$

where $E^{\prime}$ is an Eisenstein series defined as

$$
E^{\prime}\left(g^{\prime}, s\right)=\sum_{P^{\prime}(F) \backslash G^{\prime}(F)} f^{\prime}(\gamma g, s)
$$


Just as before (see [JZ]), we have an expression

$$
\begin{aligned}
I^{\prime \text { cusp }}(s)= & \sum_{\beta \in F-\{0,1\}} \Omega^{\prime}\left(\Phi^{\prime}, \beta, s\right) \\
& +\sum_{\beta \in F^{\times} / F^{\times 2}} \Omega^{\prime \infty}\left(\Phi^{\prime}, \beta, s\right) \\
& +I^{\prime u n i}\left(\Phi^{\prime}, s\right) \\
& +\sum_{i} I_{i}^{\prime}(s)
\end{aligned}
$$

where

$$
\begin{aligned}
\Omega^{\prime}\left(\Phi^{\prime}, \beta, s\right) & =\int \Phi^{\prime}\left[g^{\prime-1}\left(\begin{array}{ll}
1 & \beta \\
1 & 1
\end{array}\right) z g^{\prime}\right] \eta(z) f^{\prime}\left(g^{\prime}, s\right) d g^{\prime} \\
\Omega^{\prime \infty}\left(\Phi^{\prime}, \beta, s\right) & =\int \Phi^{\prime}\left[g^{\prime-1}\left(\begin{array}{ll}
0 & \beta \\
1 & 0
\end{array}\right) z g^{\prime}\right] \eta(z) f^{\prime}\left(g^{\prime}, s\right) d g^{\prime} \\
I^{\prime u n i}\left(\Phi^{\prime}, s\right) & =\int \Phi^{\prime}\left[g^{\prime-1}\left(\begin{array}{ll}
1 & 0 \\
1 & 1
\end{array}\right) z g^{\prime}\right] \eta(z) f^{\prime}\left(g^{\prime}, s\right) d g^{\prime}
\end{aligned}
$$

and $I_{i}^{\prime}, 1 \leqslant i \leqslant 3$, is defined below.

We introduce as before a Schwartz-Bruhat function $\phi^{\prime}$ on $F_{\mathbb{A}}$,

$$
\phi^{\prime}(x)=\int \Phi^{\prime}\left[k^{-1} z\left(\begin{array}{ll}
1 & x \\
0 & 1
\end{array}\right) k\right] \eta(z) d^{\times} z d k,
$$

and the scalar part of the intertwining operator

$$
\int f^{\prime}(w n, s) d n=m^{\prime}(s)
$$

Then

$$
I^{\prime u n i}\left(\Phi^{\prime}, s\right)=m^{\prime}(s) \int \phi^{\prime}(t)|t|^{s} d^{\times} t .
$$

The remaining integrals are

$$
\begin{aligned}
& I_{1}^{\prime}(s)=\int_{F_{a}^{\times}} \hat{\phi}^{\prime}(t)|t|^{s} d^{\times} t \\
& I_{2}^{\prime}(s)=-\frac{1}{2} \sum_{\omega^{2}=\eta} \int \Phi^{\prime}\left[k^{-1} n_{1}\left(\begin{array}{ll}
0 & 1 \\
t & 0
\end{array}\right) z n_{2} k\right] \omega(t)|t|^{(s+1) / 2} d^{\times} t d n_{1} d n_{2} d k \eta(z) d^{\times} z .
\end{aligned}
$$


We also define

$$
K^{\prime w h i}\left(g_{1}, g_{2}\right)=\int_{F_{\mathrm{a}} / F} K^{\prime e i s}\left(n_{1} g_{1}, n_{2} g_{2}\right) \psi\left(x_{1}\right) \psi\left(x_{2}\right) d n_{1} d n_{2}
$$

Then

$$
I_{3}^{\prime}(s)=\int K^{\prime w h i}\left[\left(\begin{array}{ll}
t & 0 \\
0 & 1
\end{array}\right) k,\left(\begin{array}{ll}
t & 0 \\
0 & 1
\end{array}\right) k\right]|t|^{s-1} d^{\times} t d k
$$

5.6. We now compare the two formulas we have obtained. We assume that $\Phi$ and $\Phi^{\prime}$ are products of local functions. At a place $v$ of $F$ which is inert in $E$, we assume that $\Phi_{v}$ and $\Phi_{v}^{\prime}$ have matching orbital integrals. At a place $v$ of $f$ which splits in $E$, we can identify the variety $S_{v}$ to $G_{v}^{\prime}$ and then we take $\Phi_{v}=\Phi_{v}^{\prime}$. The functions $f_{\varepsilon}$ are also taken to be product of local functions. At a place $v$ which is inert in $E$, we take

$$
\int_{H_{\varepsilon v}^{0}} f_{\varepsilon, v}(h g) d h=\Phi_{v}\left(g^{*} \varepsilon g\right) .
$$

At a place $v$ which splits into two places $v_{1}, v_{2}$ of $E$, we may identify $F_{v_{i}}$ to $F_{v}$. Then $S_{v}$ is the set of pairs $\left(s_{1}, s_{2}\right)$ with $s_{1} \in G L\left(2, F_{v}\right)$ and

$$
s_{2}=w^{t} s_{1} w
$$

Then we take

$$
\Phi_{v}\left(s_{1}, s_{2}\right)=\Phi_{v}^{\prime}\left(s_{1}\right)
$$

The image of $\varepsilon$ in $S_{v}$ is $\left(\varepsilon_{1}, \varepsilon_{2}\right)$ and the corresponding group $H_{\varepsilon v}^{0}$ is the set of pairs $\left(h_{1}, h_{2}\right)$ with

$$
h_{2}=w^{t} \varepsilon_{1}^{-1 t} h_{1}^{-1 t} \varepsilon_{1} w
$$

We demand that

$$
\int f_{\varepsilon v_{1}}\left(h_{1} g_{1}\right) f_{\varepsilon v_{2}}\left(h_{2} g_{2}\right) d h_{1}=\Phi_{v}^{\prime}\left(w^{t} g_{2} w \varepsilon_{1} g_{1}\right)
$$

that is,

$$
\int f_{\varepsilon v_{1}}\left(h_{1} g_{1}\right) f_{\varepsilon v_{2}}\left(h_{2}\right) d h_{1}=\Phi_{v}^{\prime}\left(\varepsilon_{1} g_{1}\right)
$$


If $\Phi_{v}^{\prime}$ is a Hecke function and $\varepsilon_{1}$ is in $K_{v}^{\prime}$, and $f_{\varepsilon v_{i}}$ are Hecke functions, this relation simplifies. Indeed, since $h$ and $h$ have the same double coset for the maximal compact of $G L\left(2, F_{v}\right)$, we get

$$
\int f_{\varepsilon v_{1}}(h g) f_{\varepsilon v_{2}}\left(h^{-1}\right) d h=\Phi_{v}^{\prime}(g) .
$$

In what follows, we will assume that, for a finite place $u$ of $F$ which splits in $E$, the function $\Phi_{u}^{\prime}$ and the functions $f_{\varepsilon u_{1}}, f_{\varepsilon u_{2}}$ are supercuspidal. Thus in the above discussion the kernels $K^{e i s}, K^{s p e}, K^{\prime e i s}, K^{\prime s p e}$ are zero. Moreover, the integrals $I_{2}(s)$, $I_{3}(s)$ and $I_{2}^{\prime}(s), I_{3}^{\prime}(s)$ are 0 .

The formal computations simplify. For $I^{\text {cusp }}(s)$ for instance, we find directly that

$$
I_{\Phi}^{\text {cusp }}(s)=\sum_{\beta \in F-\{0,1\}} \Omega(\Phi, \beta, s)+\sum_{\beta \in F^{\times} / F^{\times 2}} \Omega^{\text {ז }}(\Phi, \beta, s)+I^{u n i}(\Phi, s)+I_{1}(\Phi, s)
$$

where

$$
I_{1}(\Phi, s)=\left|t_{0}\right|^{1 / 2} \int_{F_{\mathrm{A}}^{\times} / F^{\times}}\left[\sum_{\beta \in F} \phi\left(t^{-1} \beta\right)\right]|t|^{s-1} d^{\times} t .
$$

However, because of the cuspidality condition, we find

$$
\int_{F_{\mathrm{A}}} \Phi\left[g^{*}\left(\begin{array}{ll}
1 & x \\
0 & 1
\end{array}\right) g\right] d x=0
$$

Thus the integral of $\phi$ vanishes, and we can write also

$$
I_{1}(s)=\left|t_{0}\right|^{1 / 2} \int_{F_{\wedge}^{\times} / F^{\times}}\left[\sum_{\beta \in F} \phi\left(t^{-1} \beta\right)-\int \phi\left(t^{-1} x\right) d x\right] \eta(t)|t|^{s-1} d^{\times} t .
$$

Thus $I_{1}(s)$ has the same expression as before.

To justify our formal computation, we need only show that in the above expression for $I^{\text {cusp }}$ all the integrals and sums converge absolutely. The above expression for $I_{1}(s)$ converges absolutely for $\Re s>1$. Similarly, the expression for $I^{\text {uni }}$ converges absolutely for $\mathfrak{R} s>1$. To prove the convergence of $\Omega(\Phi, \sigma, s)$ we proceed as in the local case, that is, we write it as a Tate integral over the multiplicative group of an algebra $L$. The trace of the matrix in the integral for $\Omega(\Phi, \beta, s)$ is $2 z$ while its determinant is $z^{2}(1-\beta)$. Thus $z^{2}(1-\beta)$ remains in a compact set of the idèles while $z$ remains in a compact set of the adèles. The first condition implies in particular that $|z|$ is in a compact set of $\mathbb{R}^{\times}$. The second condition implies then that $z$ is in a compact set of the idèles. In turn, this implies that $1-\beta$ is in a compact set of the idèles and hence in a finite set. Thus $\beta$ takes only finitely many values. The determinant of the matrix in $\Omega^{\infty}(\Phi, \beta, s)$ is $-z^{2} \beta$. It remains in a fixed compact set of the idèles. This implies that $\beta$ takes only finitely many values (modulo $F^{\times 2}$ ). Hence our formal computation is justified, for $\mathfrak{R} s>1$. 
Similarly, we find

$$
\begin{aligned}
I^{\prime c u s p}(s)= & \sum_{\beta \in F-\{0,1\}} \Omega^{\prime}\left(\Phi^{\prime}, \beta, s\right) \\
& +\sum_{\beta \in F^{\times} / F^{\times 2}} \Omega^{\prime \infty}\left(\Phi^{\prime}, \beta, s\right) \\
& +I^{\prime u n i}\left(\Phi^{\prime}, s\right) \\
& +I_{1}^{\prime}(s)
\end{aligned}
$$

for $\mathfrak{R} s>1$.

5.7 .

THEOREM 5.2. Under the previous assumptions (in particular, the condition of cuspidality at a place $u$ ), we have for all $s$

$$
\left|t_{0}\right|^{-1 / 2} L\left(s, 1_{F}\right) I^{c u s p}(s)=L(s, \eta) I^{\prime c u s p}(s) .
$$

Proof. We claim that for any place $v$ of $F$

$$
\left|t_{v 0}\right|^{-1 / 2} L\left(s, 1_{v}\right) \Omega\left(\Phi_{v}, \beta, s\right)=L\left(s, \eta_{v}\right) \Omega^{\prime}\left(\Phi_{v}^{\prime}, \beta, s\right) .
$$

Indeed, this is the condition of matching orbital integrals if $v$ is inert. Suppose $v$ splits; then $t_{v 0}=1$ by choice, and the character $\eta_{v}$ is trivial and the functions $\Phi_{v}, \Phi_{v}^{\prime}$ identical. The equality is thus trivial. Multiplying these equalities together, we obtain

$$
\left|t_{0}\right|^{-1 / 2} L\left(s, 1_{F}\right) \Omega(\Phi, \beta, s)=L\left(s, \eta_{v}\right) \Omega^{\prime}\left(\Phi_{v}^{\prime}, \beta, s\right)
$$

Similarly,

$$
\left|t_{0}\right|^{-1 / 2} L\left(s, 1_{F}\right) \Omega^{\infty}(\Phi, \beta, s)=L\left(s, \eta_{v}\right) \Omega^{\prime \infty}\left(\Phi^{\prime}, \beta, s\right) .
$$

To complete the proof we need to show that

$$
\begin{gathered}
\left|t_{0}\right|^{-1 / 2} L\left(s, 1_{F}\right) I^{u n i}(\Phi, s)=L(s, \eta) I^{\prime u n i}\left(\Phi^{\prime}, s\right), \\
\left|t_{0}\right|^{-1 / 2} L\left(s, 1_{F}\right) I_{1}(\Phi, s)=L(s, \eta) I_{1}^{\prime}\left(\Phi^{\prime}, s\right) .
\end{gathered}
$$

Since $m(s)=\left|t_{0}\right|^{s} m^{\prime}(s)$, the first relation is equivalent to

$$
\left|t_{0}\right|^{s-1 / 2} L\left(s, 1_{F}\right) \int \phi(t)|t|^{s} \eta(s) d^{\times} t=L(s, \eta) \int \phi^{\prime}(t)|t|^{s} d^{\times} t .
$$


The functions $\phi$ and $\phi^{\prime}$ are products of local functions. We claim that, for any place $v$ of $F$,

$$
\left|t_{0 v}\right|^{s-1 / 2} L\left(s, 1_{v}\right) \int \phi_{v}(t)|t|^{s} \eta(s) d^{\times} t=L\left(s, \eta_{v}\right) \int \phi_{v}^{\prime}(t)|t|^{s} d^{\times} t .
$$

Indeed, if $v$ is inert, this is a consequence of the local theory. If $v$ is split, this equality is trivial. The global equality follows.

Now we have

$$
\left|t_{0}\right|=\frac{\left|D_{F}\right|^{2}}{\left|D_{E}\right|}
$$

Using the functional equation of the $L$-functions, we see the global identity just obtained reads

$$
L\left(1-s, 1_{F}\right) \int \phi(t)|t|^{s} \eta(t) d^{\times} t=L(1-s, \eta) \int \phi^{\prime}(t)|t|^{s} d^{\times} t,
$$

or changing $s$ to $1-s$,

$$
L\left(s, 1_{F}\right) \int \phi(t)|t|^{1-s} \eta(t) d^{\times} t=L(s, \eta) \int \phi^{\prime}(t)|t|^{1-s} d^{\times} t .
$$

Using the global Tate functional equation, we find

$$
L\left(s, 1_{F}\right) \int \hat{\phi}(t)|t|^{s} \eta(s) d^{\times} t=L(s, \eta) \int \hat{\phi}^{\prime}(t)|t|^{s} d^{\times} t .
$$

Now

$$
\begin{aligned}
\left|t_{0}\right|^{-1 / 2} L\left(s, 1_{F}\right) I_{1}(s) & =\left|t_{0}\right|^{-1 / 2} L\left(s, 1_{F}\right)\left|t_{0}\right|^{1 / 2} \int \hat{\phi}(t)|t|^{s} \eta(t) d^{\times} t \\
& =L\left(s, 1_{F}\right) \int \hat{\phi}(t)|t|^{s} \eta(t) d^{\times} t \\
& =L(s, \eta) \int \hat{\phi}^{\prime}(t)|t|^{s} d^{\times} t \\
& =L(s, \eta) I_{1}^{\prime}(s) .
\end{aligned}
$$

This concludes the proof of the theorem. 
5.8. We remark that the identity of the theorem is true for any normalization of the Eisenstein series $E^{\prime}(g, s)$ provided the Eisenstein series $E(g, s)$ verifies

$$
E(g, s)=E^{\prime}(\xi(g), s)
$$

We choose $E^{\prime}$ in such a way that its residue at 1 is the constant function equal to 1. We write

$$
E(g, s)=\frac{1}{s-1}+\theta(g)+(s-1) O(1)
$$

We evaluate the residues of the two sides of our identity at $s=1$. The right-hand side has a pole of order at most 1 . Its residue is simply

$$
L(1, \eta) \int K^{\prime c u s p}\left(g^{\prime}, g^{\prime}\right) d g^{\prime}
$$

that is, apart from the factor $L(1, \eta)$, it is the standard trace formula. The left-hand side must have a simple pole at $s=1$. This shows that $I^{\text {cusp }}$ is holomorphic at $s=1$, or what amounts to the same,

$$
\int K^{\text {cusp }}(h) d h=0
$$

The residue of the left-hand side is thus

$$
\left|t_{0}\right|^{-1 / 2} \operatorname{Res}_{s=1} L\left(s, 1_{F}\right) \int K^{c u s p}(h) \theta(h) d h .
$$

More explicitly,

$$
\left|t_{0}\right|^{-1 / 2} \operatorname{Res}_{s=1} L\left(s, 1_{F}\right) \sum_{\varepsilon} \int K_{\varepsilon}^{\text {cusp }}\left(h_{\varepsilon}, h\right) \theta(h) d_{\varepsilon} d h .
$$

We have thus proved the weighted trace formula announced in the introduction.

5.9. We now state some consequences of our formula. Let $\pi$ be a cuspidal automorphic representation of $G^{\prime}$, with a supercuspidal component at $u$, and central character $\eta$. The representation $\pi \otimes \eta$ gives the same contribution to our residual trace formula. The sum of these contributions is equal to the contribution of the base change $\Pi$ of $\pi$. It follows that this contribution is nonzero. Thus there must be an $\varepsilon$ such that $\Pi$ is distinguished with respect to $H_{\varepsilon}$. Moreover, there must be a $\phi$ in 
the space of $\Pi$ such that the integral

$$
\int \phi(h) \theta(h) \eta(h) d h
$$

is nonzero. Of course this result could be obtained directly in terms of the relevant $L$-functions. It is likely that similar results can be obtained for any symmetric space.

5.10. We comment on what should happen when the assumption of cuspidality at one place is removed. The kernel $K_{\varepsilon}^{e i s}$ is integrable in the first variable on $H_{\varepsilon}$; on the other hand, $K^{\text {eis }}$ is itself an integral over a certain set of quasi characters. The double integral does not converge when $\varepsilon=1$; when one interchanges the order of integration, one gets a continuous sum, i.e. a convergent integral over a set of quasi characters and a discrete sum. If $\varepsilon \neq 1$, then one gets only a continuous sum. Thus the term $I_{3}(s)$ is the sum of a continuous part and a discrete part. The discrete part should match the contribution of the dihedral representations on $G^{\prime}$. The continuous sum should be equal to the integral $I_{3}^{\prime}(s)$. The terms $I_{2}$ and $I_{2}^{\prime}$ should be equal.

6. The second trace formula. We now explain how to modify the previous constructions to obtain the second trace formula mentioned in the introduction.

We consider first the local situation: $E$ is an extension of a nonarchimedean field $F$ of odd residual characteristic. The notations $f^{\prime}$ and $\tau_{0}$ have the same meaning as before. However, we need to replace $\tau_{0}$ by its inverse. Thus we set

$$
\tau_{1}=\tau_{0}^{-1}
$$

We define an isomorphism

$$
\xi_{1}(g)=\left(\begin{array}{cc}
\sqrt{\tau_{1}} & 0 \\
0 & 1
\end{array}\right) g\left(\begin{array}{cc}
\sqrt{\tau_{1}^{-1}} & 0 \\
0 & 1
\end{array}\right)
$$

of $H / Z$ onto $G^{\prime} Z / Z$. We set

$$
f_{1}(g)=f^{\prime}\left(\xi_{1}(g)\right)
$$

For $\Phi$ smooth of compact support on $S$, we now set

$$
\Omega(\Phi, \sigma, s)=\int \Phi\left(h^{-1} \sigma z h\right) f_{1}(h, s) d h d^{\times} z .
$$

For $\Phi^{\prime}$ smooth of compact support on $G^{\prime}$, we set

$$
\Omega^{\prime}\left(\Phi^{\prime}, \sigma, s\right)=\int \phi^{\prime}\left(g^{-1} \sigma z g\right) f^{\prime}(g, s) \eta(\operatorname{det} g) d g \eta(z) d^{\times} z .
$$


We say that $\Phi$ and $\Phi^{\prime}$ have "matching orbital integrals" if

$$
\left|\tau_{1}\right|^{-1 / 2} L(s, \eta) \Omega(\Phi, \sigma, s)=\eta\left(\beta_{1}\right) L\left(s, 1_{F}\right) \Omega^{\prime}\left(\Phi^{\prime}, \sigma, s\right)
$$

for all

$$
\sigma=\left(\begin{array}{cc}
\alpha & \beta_{2} \\
\beta_{1} & \alpha
\end{array}\right)
$$

with $\beta_{1} \beta_{2} \neq 0$. As before, we introduce the notations $\Omega(\Phi, \beta, s), \Omega^{\infty}(\Phi, \beta, s)$ and the similar notations for $\Omega^{\prime}$. Just as before, we have the following fundamental lemma:

Proposition 6.1. If the extension $E$ is unramified, then the functions $\Phi_{0}$ and $\Phi_{0}^{\prime}$ have matching orbital integrals. More generally, if $\Phi$ is a function of compact support on $S$ invariant under $K$ and $\Phi^{\prime}$ is a function of compact support on $G^{\prime}$ bi-invariant under $K^{\prime}$ which corresponds to $\Phi$, then $\Phi$ and $\Phi^{\prime}$ have matching orbital integrals.

Proof. Recall that $\tau_{1}$ is then any unit which is not a square. With the notation of Section 3, it will suffice to show that $\Phi_{n}$ and $\Phi_{2 n}^{\prime}$ have matching orbital integrals.

We get as before that $\Omega\left(\Phi_{n}, \beta, s\right)$ and $\Omega^{\prime}\left(\Phi_{2 n}^{\prime}, \beta, s\right)$ are zero unless

$$
|1-\beta|=|\gamma|^{2}
$$

and then

$$
\begin{aligned}
\Omega\left(\Phi_{n}, \beta, s\right) & =|\gamma|^{s} q^{n s} A\left(\frac{\beta \varpi^{2 n}}{\gamma^{2}}, X\right), \\
\Omega^{\prime}\left(\Phi_{2 n}, \beta, s\right) & =|\gamma|^{s} q^{n s} A\left(\frac{\beta \varpi^{2 n}}{\gamma^{2} \tau_{1}},-X\right) .
\end{aligned}
$$

Then our assertion follows from the functional equation satisfied by the function $A$.

Next, we have the following result:

Proposition 6.2. Given $\Phi$, there is a $\Phi^{\prime}$ with matching orbital integrals and conversely.

Proof. We only discuss the asymptotic behavior of our integrals when $\beta$ tends to 0 and check they are compatible.

We first study the asymptotic behavior of the integrals $\Omega(\Phi, \beta, s)$ when $\beta$ tends to 0 . As before, we will denote by $I(\Phi, s)$ the integral corresponding to $\beta=0$.

$$
I(\Phi, s)=\int \Phi\left[h^{-1} z\left(\begin{array}{ll}
1 & 0 \\
1 & 1
\end{array}\right) h\right] f(h, s) d h
$$


We consider also the integral

$$
J(\Phi, s)=\int \Phi\left[h^{-1} z\left(\begin{array}{ll}
1 & 1 \\
0 & 1
\end{array}\right) h\right] f(h, s) d h d^{\times} z,
$$

the integral over the quotient $Z N_{H} \backslash H$.

It will be convenient to introduce the following function $\phi$ on $F$ :

$$
\phi(x)=\int \Phi\left[k^{-1} z\left(\begin{array}{ll}
1 & x \\
0 & 1
\end{array}\right) k\right] d k d^{\times} z .
$$

Thus $\phi$ is an arbitrary Schwartz-Bruhat function on $F$ such that

$$
\phi(u x)=\phi(x)
$$

for all $u \in F$ with $|u|=1$. In terms of $\phi$,

$$
\begin{aligned}
& I(\Phi, s)=m_{1}(s) \int \phi(t)|t|^{s} \eta(t) d^{\times} t, \\
& J(\Phi, s)=\int \phi(t)|t|^{1-s} d^{\times} t .
\end{aligned}
$$

The first integral converges for $\mathfrak{R} s>0$ and the second for $\mathfrak{R}<1$. These distributions determine the "Shalika germs" of the orbital integrals. Namely, let $A>0$ be a constant and set for $\beta$ an integer

$$
C_{1}(\beta, s)=\int_{|x| \leqslant A}\left(\left|\beta-x^{2}\right|^{s-1}-\left|x^{2}\right|^{s-1}\right) d x
$$

where

$$
x=\frac{u}{\sqrt{\tau_{1}}}, \quad u \in F, d x=d u .
$$

Proposition 6.3. Given $\Phi$, for $|\beta|$ sufficiently small and all s with $0<\Re s<1$,

$$
\Omega(\Phi, \beta, s)=I(\Phi, s)+C_{1}(\beta, s) J(\Phi, s) .
$$

The proof is the same as before.

6.1. We consider similarly the integrals $\Omega^{\prime}$. Recall the integral

$$
\Omega^{\prime}\left(\Phi^{\prime}, \beta, s\right)=\int \Phi^{\prime}\left[g^{-1} z \sigma g\right] f(g, s) \eta(\operatorname{det} g) d g \eta(z) d^{\times} z
$$


where

$$
\sigma=\left(\begin{array}{ll}
1 & \beta \\
1 & 1
\end{array}\right)
$$

As before, we consider the orbital integral corresponding to $\beta=0$ :

$$
I^{\prime}\left(\Phi^{\prime}, s\right)
$$

Similarly, we define

$$
J^{\prime}\left(\Phi^{\prime}, s\right)=\int \Phi^{\prime}\left[g^{\prime-1} z\left(\begin{array}{ll}
1 & 1 \\
0 & 1
\end{array}\right) g^{\prime}\right] f^{\prime}\left(g^{\prime}, s\right) \eta\left(\operatorname{det} g^{\prime}\right) d g^{\prime} \eta(z) d^{\times} z .
$$

As before, we introduce the following function $\phi^{\prime}$ on $F$ :

$$
\phi^{\prime}(x)=\int \Phi^{\prime}\left[k^{-1} z\left(\begin{array}{cc}
1 & x \\
0 & 1
\end{array}\right) k\right] \eta(\operatorname{det} k) d k \eta(z) d^{\times} z .
$$

Thus $\phi^{\prime}$ is an arbitrary Schwartz-Bruhat function on $F$ such that

$$
\phi^{\prime}(u x)=\eta(u) \phi^{\prime}(x)
$$

for all $u \in F$ with $|u|=1$. In terms of $\phi^{\prime}$, we have

$$
\begin{aligned}
& I^{\prime}\left(\Phi^{\prime}, s\right)=m^{\prime}(s) \int \phi^{\prime}(t) \eta(t)|t|^{s} d^{\times} t \\
& J^{\prime}\left(\Phi^{\prime}, s\right)=\int \phi^{\prime}(t) \eta(t)|t|^{1-s} d^{\times} t .
\end{aligned}
$$

We define

$$
C_{1}^{\prime}(\beta, s)=\int_{|x| \leqslant A}\left(\eta\left(\beta-x^{2}\right)\left|\beta-x^{2}\right|^{s-1}-\eta\left(-x^{2}\right)\left|x^{2}\right|^{s-1}\right) d x .
$$

As before, this does not depend on the value of $A$, provided $|\beta|$ is small enough and we have the following proposition:

Proposition 6.4. Given $\Phi^{\prime}$, for $|\beta|$ sufficiently small and all s with $\mathfrak{R} s>0$,

$$
\Omega^{\prime}\left(\Phi^{\prime}, \beta, s\right)=I^{\prime}\left(\Phi^{\prime}, s\right)+C_{1}^{\prime}(\beta, s) J^{\prime}\left(\Phi^{\prime}, s\right)
$$

The proof is the same as before. 
6.2. The first step and the only one we discuss in matching the orbital integrals is the following lemma:

LEMMa 6.5. Given $\Phi$, there is $\Phi^{\prime}$ such that for all s and all $\beta$ sufficiently small,

$$
\left|\tau_{1}\right|^{-1 / 2} \Omega(\Phi, \beta, s) L(s, \eta)=\Omega^{\prime}\left(\Phi^{\prime}, \beta, s\right) L\left(s, 1_{F}\right) .
$$

Proof. It suffices to show that, given $\Phi$, there is $\Phi^{\prime}$ with

$$
\begin{gathered}
\left|\tau_{1}\right|^{-1 / 2} I(\Phi, s) L(s, \eta)=I^{\prime}\left(\Phi^{\prime}, s\right) L\left(s, 1_{F}\right) \\
\left|\tau_{1}\right|^{-1 / 2} C_{1}(\beta, s) J(\Phi, s) L(s, \eta)=C_{1}^{\prime}(\beta, s) J^{\prime}\left(\Phi^{\prime}, s\right) L\left(s, 1_{F}\right) .
\end{gathered}
$$

Since

$$
m_{1}(s)=m^{\prime}(s)\left|\tau_{1}\right|^{s}
$$

the relation (78) reads

$$
\eta(-1)\left|\tau_{1}\right|^{s-1 / 2} \int \phi(x)|x|^{s} d^{\times} x L(s, \eta)=\int \phi^{\prime}(x) \eta(x)|x|^{s} d^{\times} x L\left(s, 1_{F}\right) .
$$

As before, given $\phi$, there is $\phi^{\prime}$ such that this relation is satisfied. Then the relation (79) will be a consequence of the following identity that we state as a lemma:

LEMMA 6.6. With the above notation,

$$
\frac{L\left(s, 1_{F}\right) C_{1}^{\prime}(\beta, s)}{L\left(1-s, 1_{F}\right)}=\eta(-1)\left|\tau_{1}\right|^{s-1} \frac{L(s, \eta) C_{1}(\beta, s)}{L(1-s, \eta)}
$$

provided $|\beta|$ is small enough.

Indeed, we have

$$
\begin{aligned}
& C_{1}(\beta, s)=\left|\tau_{0}\right|^{s-1} C^{\prime}\left(\frac{\beta}{\tau_{0}}, s\right) \\
& C_{1}^{\prime}(\beta, s)=\eta(-1)\left|\tau_{0}\right|^{s-1} C\left(\frac{\beta}{\tau_{0}}, s\right)
\end{aligned}
$$

Thus the above equation is equivalent to the corresponding equation for the pair $\left(C, C^{\prime}\right)$.

6.3. Now we let $E / F$ be a quadratic extension of number fields. As before, we assume that every real place of $F$ splits in $E$ and that every finite place of even residual characteristic splits in $E$. For each place $v$ of $F$ inert in $E$ we choose an 
element $t_{1, v}$ such that

$$
E_{v}=F_{v}\left(\sqrt{t_{1, v}}\right)
$$

If $v$ is unramified in $E$, we take $t_{1, v}$ of module 1 ; if $v$ is ramified, we take for $t_{1, v}$ the inverse of a uniformizer of $F_{v}$. If $v$ splits in $E$, we take $t_{v}=1$. We set

$$
t_{1}=\prod t_{1, v}
$$

Conjugacy by

$$
\left(\begin{array}{cc}
\sqrt{t_{1, v}} & 0 \\
0 & 1
\end{array}\right)
$$

defines for each inert place $v$ an isomorphism

$$
\xi_{1, v}: H\left(F_{v}\right) \rightarrow G^{\prime}\left(F_{v}\right) Z\left(F_{v}\right) .
$$

At a split place we have a natural isomorphism. As before, we define an isomorphism

$$
\xi_{1}: H\left(F_{\mathbb{A}}\right) \rightarrow G^{\prime}\left(F_{\mathbb{A}}\right) Z\left(F_{\mathbb{A}}\right) .
$$

We use it to transport $K^{\prime} U$ and $f^{\prime}$ to $H$. We denote by $K_{H}$ and $f_{1}$ the corresponding maximal subgroup and function. Thus

$$
f_{1}(h, s)=|t|^{s}, \quad \text { if } h=\left(\begin{array}{cc}
t z & x \\
0 & z
\end{array}\right) k .
$$

Then

$$
E_{1}(h, s)=\sum_{\gamma \in P_{H}(F) \backslash H(F)} f_{1}(\gamma h, s) .
$$

On $G^{\prime}\left(F_{\mathbb{A}}\right) / Z^{\prime}\left(F_{\mathbb{A}}\right)$, we use the same measure $d g^{\prime}$ as before. We transport this measure to $H\left(F_{\mathrm{A}}\right) / Z\left(F_{\mathrm{A}}\right)$. The measure need not be the same as the one we have used to define the kernels in terms of $\Phi$. Given a smooth function of compact support $\Phi$ on $S\left(F_{\mathbb{A}}\right)$, we define as before a cuspidal kernel $K^{\text {cusp }}$ and the integral

$$
I^{c u s p}(s)=\int_{Z\left(F_{\mathrm{A}}\right) H(F) \backslash H\left(F_{\mathrm{A}}\right)} K^{\text {cusp }}(h) E_{1}(h, s) d h .
$$

Similarly, given a smooth function of compact support $\Phi^{\prime}$ on $G^{\prime}\left(F_{\mathbb{A}}\right)$, we define the cuspidal kernel $K^{\prime c u s p}$ and the integral

$$
I^{\prime c u s p}(s)=\int K^{\prime c u s p}\left(g^{\prime}, g^{\prime}\right) \eta\left(\operatorname{det} g^{\prime}\right) E^{\prime}(g, s) d g^{\prime}
$$


where $E^{\prime}$ is the Eisenstein series defined as

$$
E^{\prime}\left(g^{\prime}, s\right)=\sum_{P^{\prime}(F) \backslash G^{\prime}(F)} f^{\prime}(\gamma g, s) .
$$

We assume that $\Phi$ and $\Phi^{\prime}$ are product of local functions. At a place $v$ of $F$ inert in $E$, we assume that $\Phi_{v}$ and $\Phi_{v}^{\prime}$ have matching orbital integrals in the sense of the present section. At a place $v$ of $f$ which splits in $E$, we can identify the variety $S_{v}$ to $G_{v}^{\prime}$ and then take $\Phi_{v}=\Phi_{v}^{\prime}$. The functions $f_{\varepsilon}$ are chosen as before. Then we have the following result.

THEOREM 6.7. Under the previous assumptions (in particular, the condition of cuspidality at a place $u$ ), we have for all $s$

$$
\left|t_{1}\right|^{-1 / 2} L(s, \eta) I^{c u s p}(s)=L\left(s, 1_{F}\right) I^{\prime c u s p}(s)
$$

The proof is the same as before.

6.4. If we normalize the Eisentein series as before and take the residue at $s=1$ of both sides of our identity, we obtain

$$
\begin{aligned}
& \left|t_{1}\right|^{-1 / 2} L(1, \eta) \sum_{\varepsilon} \int K_{\varepsilon}\left(h_{\varepsilon}, k\right) d h_{\varepsilon} d h \\
& \quad=\left.\operatorname{Res} L(s, 1)\right|_{s=1} \int K_{\text {cusp }}^{\prime}\left(g^{\prime}, g^{\prime}\right) \theta\left(g^{\prime}\right) \eta\left(\operatorname{det} g^{\prime}\right) d g^{\prime}
\end{aligned}
$$

This is the other weighted trace formula mentioned in the introduction.

\section{REFERENCES}

[A] T. AsAl, On certain Dirichlet series associated with Hilbert modular forms and Rankin's method, Math. Ann. 226 (1977), 81-94.

[F] Y. FliCKER, On distinguished representations, J. Reine Angew. Math. 418 (1991), 139-172.

[FH] Y. FLICKER AND J. HAKIM, Quaternionic distinguished representations, to appear in Amer. J. Math.

[Ha] J. HAKIM, Distinguished p-adic representations, Duke Math. J. 62 (1991), 1-22.

[HLR] G. HARDER, R. LANGLANDS, AND M. RAPOPORT, Algebraische Zyklen auf Hilbert-Blumenthal Flächen, J. Reine Angew. Math. 366 (1986), 53-120.

[Ho] R. Howe, Theta Series and Invariant Theory, Proc. Sympos. Pure Math. 33, Amer. Math. Soc., Providence, 1979, 275-306.

[I1] H. IwANIEC, Fourier coefficients of modular forms of half integral weight, Invent. Math. 87 (1987), 385-401.

[I2] On Waldspurger's theorem, Acta Arith. 49 (1987), 205-212.

[J1] H. JACQUET, Automorphic forms on GL(2) II, Lecture Notes in Math. 278, Springer-Verlag, New York, 1972.

[J2] - On the nonvanishing of some L-functions, Proc. Indian Acad. Sci. Math. Sci. 97 (1987), $117-155$. 
[J3] Kloosterman integrals for GL(3), to appear in Canad. J. Math.

[JL] H. JACQUET AND K. F. LAI, A relative trace formula, Compositio Math. 54 (1985), 243-310.

[JR] H. JACQUET AND S. RALLIS, Kloosterman integrals for skew symmetric matrices, Pacific J. Math. 154 (1992), 265-283.

[JY1] Une remarque sur le changement de base quadratique, C.R. Acad. Sci. Paris Sér. I Math. 311 (1990), 671-676.

[JY2] H. JACQUET AND Y. YANGBO, Relative Kloosterman integrals for GL(3), Bull Soc. Math. France 120 (1992), 263-295.

[JZ] H. JACQUET AND D. ZAGIER, Eisenstein series and the Selberg trace formula II, Trans. Amer. Math. Soc. 300 (1987), 1-47.

[kL] K. F. LAI, Algebraic cycles on compact Shimura surfaces, Math. Z. 189 (1985), 593-602.

[KR] B. Kostant AND S. RALLIS, Orbits and representations associated with symmetric spaces, Amer. J. Math. 93 (1971), 275-306.

[rL] R. Langlands, Base Change for GL(2), Ann. of Math. Stud. 96, Princeton Univ. Press, Princeton, 1980.

[M] Z. MAO, Relative Kloosterman integrals for the unitary group, C.R. Acad. Sci. Paris Sér. I Math. 315 (1992), 381-386.

[R] R. RichaRdSON, Orbits, invariants, and representations associated to involutions of reductive groups, Invent. Math. 66 (1982), 287-312.

[Y] Y. YE, Kloosterman integrals and base change for GL(2), J. Reine Angew. Math. 400 (1989), 57-121.

[Z] D. ZAGIER, Modular forms associated to real quadratic fields, Invent. Math. 30 (1975), 1-46.

Jacquet: Department of Mathematics, Columbia University, New York, New York, 10027, USA LaI: School of Mathematics and Statistics, The University of Sydney, Sydney NSW 2006, Australia

Rallis: Department of Mathematics, The Ohio State University, Columbus, Ohio 43210, USA 